Pacific Journal of Mathematics

THE ISOTROPY REPRESENTATION FOR HOMOGENEOUS 


\title{
THE ISOTROPY REPRESENTATION FOR HOMOGENEOUS SIEGEL DOMAINS
}

\author{
J. E. D'Atri, J. Dorfmeister, AND Zhao YAN DA
}

This paper gives several new characterizations of symmetric domains among the class of homogeneous Siegel domains. These characterizations involve the commutativity of the algebra of invariant differential operators, the transitivity of the action of the isotropy group on the Šilov boundary, and the representation of the almost complex structure by the infinitesimal isotropy action, respectively.

Homogeneous Siegel domains (equivalently, homogeneous bounded domains) are important geometric objects to study. They are more general than Hermitian symmetric spaces. They form a special class of homogeneous Kähler manifolds which would be one of the three building blocks of an arbitrary homogeneous Kähler manifold according to a conjecture of Gindikin and Vinberg. But they have also certain properties which are typical for arbitrary homogeneous Riemannian manifolds of non-positive curvature (NC algebras).

In this paper we investigate certain relations between homogeneous Siegel domains and the three different types of homogeneous spaces mentioned above. In particular, as the main result of this paper we give five different new characterizations of symmetric domains amongst the class of homogeneous Siegel domains. So if $D$ is a homogeneous Siegel domain, $G$ the identity component of the automorphism group of $D$, and $K$ the isotropy subgroup at a point $b$ of $D$, then the following are equivalent:

(a) $D$ is symmetric

(b) The almost complex structure map on the tangent space $T_{b} D$ at $b$ is in the image of the infinitesimal isotropy representation.

(c) There exist no nontrivial $G$ invariant vector fields

(d) The algebra of $G$ invariant differential operators on $D$ is commutative

(e) The isotropy group acts transitively on the Šilov boundary

(f) There exists a vector field $X$ in the center of the isotropy algebra at the basepoint $b$ of $D \subset \mathbf{C}^{n}$ so that the differential of $X$ is invertible as a linear transformation on $\mathbf{C}^{n}$. 
Note that the following characterization is already known to be equivalent with (a) [6]; (g) All sectional curvatures of $D$ are nonpositive in the Bergman metric.

As in the proof of the equivalence of (g), substantial progress can be made on our main result using only the machinery of normal $j$-algebras. However, again as in the previous proof, we reach crucial points where it seems necessary to use the algebraic structures of Dorfmeister [7-12] and Vinberg $[30,31]$. In this case, we also use intensively the linear isotropy representation of $K$ and Lie $K$ in terms closely related to the NC algebra theory of Azencott-Wilson [1,2]. Altogether, we have to combine parts of three different, very technical and refined theories to prove our main results. As this situation is likely to arise again and as these three theories have not yet been interrelated, we include enough material to establish the relations between them. Due to the nature of these theories, it is unavoidable that this paper is also notationally difficult; however, we feel that the results justify the effort. Moreover, building on this paper, the second author described all homogeneous Kähler structures on $D[14]$ and proved a special case of the aforementioned Gindikin-Vinberg conjecture [15].

We now describe the organization of our paper. Section 3 gives the proofs of the equivalences. The proof of characterization (b) (Theorem 5) is easy and depends only on the introductory paragraph of section 2 on the isotropy representation. The proofs of characterizations (c) and (d) (Theorems 7 and 8) follow easily from Theorem 3 of $\$ 2$, which is of independent interest as a refined structure theorem for normal $j$-algebras. However, the proof of Theorem 3 is not direct. A proof may be given using only normal $j$-algebras but it would be quite computational. It is the proof of characterizations (e) and (f) (Theorem 6 with the following Remarks and Theorem 9) which finally decides the methods to be used in this paper since we could find no way of effectively computing the orbits of the isotropy group on the Šilov boundary using only normal $j$-algebras (and the problem of further analyzing the structure of the algebra of invariant differential operators in the noncommutative case also seems to require these more complicated tools, see $\$ 3.1)$. Thus our method is based firstly on Theorem 1 of $\S 2$ (implicit in the work of Azencott-Wilson [2] and similar to a result in the unpublished thesis of H. L. Williams [32]) which says that under certain technical conditions, the image of the infinitesimal isotropy representation is spanned by all skew-symmetric derivations and certain covariant derivatives, which can be determined by computations within the Lie algebra of a simply transitive group. Secondly, it is necessary to develop enough of the algebraic theory of [7-12] 
to show that the conditions of this Azencott-Wilson type theorem are satisfied to prove Theorem 3 (which in turn requires reinterpreting things in the normal $j$-algebra context), and to explicitly compute the infinitesimal isotropy representation (Theorem 4); this is done in $\S 1$ and the rest of $\S 2$. Finally, §4 contains examples which may be useful.

1. Let $D=\left\{(Z, U) \in \mathscr{V}^{\mathbf{C}} \times \mathscr{U}: \operatorname{Im} Z-F(U, U) \in \Omega\right\}$ be the homogeneous Siegel domain determined by the homogeneous regular cone (i.e. open convex and not containing any entire straight line) $\Omega$ in the real (finite dimensional) vector space $\mathscr{V}$, the complex (finite dimensional) vector space $\mathscr{U}$ and the $\Omega$-hermitian form $F: \mathscr{U} \times \mathscr{U} \rightarrow \mathscr{V}^{\mathbf{C}}=\mathscr{V} \oplus i \mathscr{V}$, [27]. Let $G$ be the identity component of the automorphism group of $D$ with Lie algebra g. Every $X \in \mathfrak{g}$ determines a real vector field $X^{*}$ on $D$ by $\left.X^{*}\right|_{p}=d /\left.d t\right|_{t=0}(\exp t X) \cdot p$ and the map $X \rightarrow X^{*}$ is real linear injective with $\left[X^{*}, Y^{*}\right]=-[X, Y]^{*}$. The superscript ${ }^{*}$ will in general denote the image of this map so, for example, the grading (see [21]) $\mathfrak{g}=\mathfrak{g}_{-1} \oplus \mathfrak{g}_{-1 / 2}$ $\oplus \mathfrak{g}_{0} \oplus \mathfrak{g}_{1 / 2} \oplus \mathfrak{g}_{1}$ gives a $\mathfrak{g}^{*}=\mathfrak{g}_{-1}^{*} \oplus \mathfrak{g}_{-1 / 2}^{*} \oplus \mathfrak{g}_{0}^{*} \oplus \mathfrak{g}_{1 / 2}^{*} \oplus \mathfrak{g}_{1}^{*}$ of the Lie algebra $g^{*}$ of vector fields (direct sums are always as vector spaces unless otherwise indicated). For $X \in \mathfrak{g}^{*}$, the preimage in $\mathfrak{g}$ will be denoted by $X^{+}$. In general, a real vector space is identified naturally with its tangent space at each point and each element $V$ of the vector space determines an obvious vector field denoted $\Delta^{V}$ (directional derivative). When this notation is used for a complex vector space, it refers to the underlying real structure.

In general, we use the notation of [12] but denote the cone by $\Omega$ (instead of $K$ ), the $\Omega$-hermitian form by $F$ (instead of $S$ ), the fundamental algebra (on the underlying vector space $\mathscr{V}$ ) by $\mathscr{L}$ (instead of $\mathscr{A}$ ), and the left multiplication in $\mathscr{L}$ by $L(X)$ (instead of $A(X)$ ). One also has the mutation multiplication operators $L_{X}(Y)=L(X Y)-[L(X), L(Y)]$ (instead of $A_{X}(Y)$ ). The Bergman kernel function $B$ is determined by a function $\eta$ (abbreviated from $\eta_{K S}$ ) by

$$
B\left(Z_{1}+U_{1}, Z_{2}+U_{2}\right)=\eta\left((2 i)^{-1}\left(Z_{1}-\bar{Z}_{2}\right)-F\left(U_{1}, U_{2}\right)\right)
$$

The identity of $\mathscr{L}$ will be a point $e$ in $\Omega$ with $\eta(e)=1$. The algebra structure on $\mathscr{L}$ and a real inner product $\sigma$ are defined on $\mathscr{V}$ (and extended C linearly to $\mathscr{V}^{\mathbf{C}}$ ) by

$$
\begin{aligned}
& \sigma(X, Y)=\left(\Delta^{X} \Delta^{Y} \log \eta\right)(e), \\
& \sigma(X Y, Z)=-\frac{1}{2}\left(\Delta^{X} \Delta^{Y} \Delta^{Z} \log \eta\right)(e), \quad X, Y, Z \in \mathscr{V} .
\end{aligned}
$$


We also have on $\mathscr{U}$ the hermitian form $\rho(U, V)=\sigma(F(U, V), e)$. If $g$ denotes the Bergman metric of $D$, then

$$
\begin{aligned}
g_{i e}\left(Z_{1}+U_{1}, Z_{2}+U_{2}\right)=2 c \operatorname{Re}\left(\frac{1}{4} \sigma\left(Z_{1}, \bar{Z}_{2}\right)+\rho\left(U_{1}, U_{2}\right)\right), \\
Z_{K} \in \mathscr{V} \mathbf{c}, U_{K} \in \mathscr{U} .
\end{aligned}
$$

Here $c$ is a positive constant and conjugation in $\mathscr{V}^{\mathbf{C}}$ is with respect to $\mathscr{V}$ as real part.

Remarks (1) The Bergman metric is Einstein with Ric $=-(1 / c) g$ for the constant $c$ above. Different normalizations are in use, e.g. $c=1$ in [3-6, 22] and $c=1 / 2$ in [18]. We will use $c=1 / 2$.

(2) $i \Omega$ is a totally geodesic submanifold of $(D, g)$. The obvious diffeomorphism $\Omega \tilde{\rightarrow} i \Omega$ then induces a Riemannian metric on $\Omega$ from $g$. With respect to the Riemannian connection, the covariant derivative of $\Delta^{Y}$ in the direction $\Delta^{X}$ at $e$ is $\left(-\Delta^{X Y}\right)_{e}$ where $X Y$ is the product in $\mathscr{L}$.

With the identification of each (real) tangent space of $\mathscr{V}^{\mathrm{C}} \times \mathscr{U}$ with $\mathscr{V}^{\mathbf{C}} \times \mathscr{U}$, we can identify a vector field on $D$ with a function $D \rightarrow \mathscr{V}^{\mathrm{C}} \times \mathscr{U}$. We use this to define vector fields

$$
\begin{aligned}
& X_{-1}[a]=((Z, U) \rightarrow(a, 0))=\Delta^{a} \quad \text { for } a \in \mathscr{V} \\
& X_{-1 / 2}[d]=((Z, U) \rightarrow(2 i F(U, d), d) \text { for } d \in \mathscr{U}
\end{aligned}
$$

and have $\mathrm{g}_{-1}^{*}=\left\{X_{-1}[a]: a \in \mathscr{V}\right\}, \mathrm{g}_{-1 / 2}^{*}=\left\{X_{-1 / 2}[d]: d \in \mathscr{U}\right\}$. For any $T \in \mathfrak{g l}(\mathscr{V})$, we always extend $T \mathbf{C}$-linearly to $\mathscr{V}^{\mathbf{C}}$ and let $\hat{T}$ denote an element of $\mathfrak{g} \mathfrak{l}(\mathscr{U}, \mathbf{C})$ for which $T(F(U, V))=F(\hat{T} U, V)+F(U, \hat{T} V)$. The Lie algebra of $\operatorname{Gl}(D)$ (the linear automorphism group of $D$ ) is $\mathrm{g}_{0} \subset$ $\{(T, \hat{T}): T \in \mathfrak{g} \mathfrak{l}(\mathscr{V})\}$ and $(T, \hat{T})^{*}$ is the function $(Z, U) \rightarrow(T Z, \hat{T} U)$. For consistency and to avoid confusion, set

$$
X_{0}(T, \hat{T})=((Z, U) \rightarrow(T Z, \hat{T} U))=(T, \hat{T})^{*} .
$$

Finally, there are vector subspaces $\mathscr{P}_{1} \subset \mathscr{V}, \mathscr{P}_{1 / 2} \subset \mathscr{U}$ so that $\mathrm{g}_{1 / 2}^{*}=$ $\left\{X_{1 / 2}[d]: d \in \mathscr{P}_{1 / 2}\right\}, \mathfrak{g}_{1}^{*}=\left\{X_{1}[a]: a \in \mathscr{P}_{1}\right\}$ where

$$
\begin{aligned}
& X_{1 / 2}[d]=((Z, U) \rightarrow(2 F(U, \phi(\bar{Z}) d), \\
&i \phi(Z) d+2 \phi(F(U, d)) U)) \\
& X_{1}[a]=\left((Z, U) \rightarrow\left(\left(L_{a}(Z)\right)(Z), \phi(Z) \phi(a) U\right)\right)
\end{aligned}
$$

and $\phi$ is defined by $\rho(\phi(X) U, V)=\sigma(F(U, V), X), X \in \mathscr{V}^{\mathbf{c}}, U, V \in \mathscr{U}$. We extensively use $[12, \S 6]$ which gives the bracket products of these vector fields (i.e. the Lie product on $\mathrm{g}^{*}$ ). Note that all these vector fields canonically extend to $\mathscr{V}^{\mathbf{C}} \times \mathscr{U}$. For

$$
X^{*}=X_{-1}[a]+X_{-1 / 2}[d]+X_{0}(T, \hat{T})+X_{1 / 2}\left[d^{\prime}\right]+X_{1}\left[a^{\prime}\right],
$$


one has $X_{l e}^{*}=\left(a-a^{\prime}+i T e, d-d^{\prime}\right)$ since $\phi(e)=I$. In particular, if $K$ is the isotropy subgroup of $G$ at $i e$ with corresponding subalgebra $f$, then

$$
\begin{aligned}
\mathfrak{F}^{*}= & \left\{X_{-1}[a]+X_{1}[a]: a \in \mathscr{P}_{1}\right\} \\
& \oplus\left\{X_{-1 / 2}[d]+X_{1 / 2}[d]: d \in \mathscr{P}_{1 / 2}\right\} \\
& \oplus\left\{X_{0}(T, \hat{T}):(T, \hat{T}) \in \mathfrak{g}_{0}, T e=0\right\} .
\end{aligned}
$$

Since $G$ is connected and $D$ is simply connected, we have that $K$ is connected. We also define a bilinear form $\langle$,$\rangle on \mathfrak{g}$ by

$$
\langle X, Y\rangle=g_{i e}\left(X^{*}, Y^{*}\right) \text {. }
$$

Following [12, §3], we have idempotents $e_{11}, \ldots, e_{q q}$ of $\mathscr{L}$ giving a Peirce decomposition $\mathscr{L}=\bigoplus_{1 \leq i \leq j \leq q} \mathscr{L}_{i j}$ where each $\mathscr{L}_{i i}$ is a formally real Jordan algebra with respect to $\sigma$ and $e=\sum e_{i l}$. To be consistent with the notation of [3-6], we will reverse the ordering of the idempotents as used in [7-12]. Thus $\mathscr{P}_{1}$ is an ideal of $\mathscr{L}_{q q}$ whose orthogonal complement in $\mathscr{L}_{q q}$ will be denoted $\mathscr{L}_{q q}^{0}$. Let

$$
\mathscr{X}=\bigoplus_{1 \leq j \leq q} \mathscr{L}_{j j} \text { and } \mathscr{X} \ominus \mathscr{P}_{1}=\bigoplus_{1 \leq j \leq q-1} \mathscr{L}_{j j} \oplus \mathscr{L}_{q q}^{0}
$$

We need the following result from [12, Corollary 3.11] (given in our new ordering)

(9) If $X \in \mathscr{L}_{j j}, Y \in \mathscr{L}_{i j}$ and $i \leq j$, then $\left(L_{X}(Y), \frac{1}{2} \phi(Y) \phi(X)\right) \in \mathrm{g}_{0}$.

Our aim now is to construct a graded subalgebra $\mathfrak{s}$ of $\mathfrak{g}_{-1} \oplus \mathfrak{g}_{-1 / 2} \oplus \mathfrak{g}_{0}$ (the Lie algebra of the affine group of $D$ ) which will have the structure of a normal $j$-algebra (cf. [28] for definition). For this, we need a complete set $\left\{d_{1}, \ldots, d_{r}\right\}$ of primitive orthogonal idempotents of $\mathscr{L}$ giving a finer Peirce decomposition. More precisely, choose the new idempotents to lie in the simple formally real summands of the spaces $\mathscr{L}_{11}, \mathscr{L}_{22}, \ldots, \mathscr{L}_{q q}^{0}, \mathscr{P}_{1}$ and indexed increasingly in that order. Note that $e=\sum d_{i}$. Let $\mathscr{L}=$ $\bigoplus_{1 \leq i \leq 1 \leq r} \mathscr{B}_{i j}$ denote the Peirce decomposition of $\mathscr{L}$ relative to $\left\{d_{1}, \ldots, d_{r}\right\}$. Then $\mathscr{B}_{i i}=\mathbf{R} d_{i}$ and $\mathscr{B}_{i j} \subset \mathscr{L}_{v w}$ whenever $d_{i} \in \mathscr{L}_{v v}, d_{j} \in \mathscr{L}_{w w}$. Motivated by [8; Theorem 5.1] and [12; Lemma 3.10] and using (9) (note the effect of the opposite ordering), one defines an element $\left(T\left(b_{i j}\right), \hat{T}\left(b_{i j}\right)\right) \in \mathfrak{g}_{0}$ for each $b_{i j} \in \mathscr{B}_{i j}$ by

$$
\begin{aligned}
& \left(T\left(b_{i j}\right), \hat{T}\left(b_{i j}\right)\right) \\
& \quad=\left\{\begin{array}{l}
\left(L_{d_{i}}\left(b_{i i}\right), \frac{1}{2} \phi\left(b_{i i}\right) \phi\left(d_{i}\right)\right)=\left(L\left(b_{i i}\right), \frac{1}{2} \phi\left(b_{i i}\right)\right) \text { if } i=j \\
\left(L_{d_{j}}\left(2 b_{i j}\right), \frac{1}{2} \phi\left(2 b_{i j}\right) \phi\left(d_{j}\right)\right) \text { if } i<j .
\end{array}\right.
\end{aligned}
$$

More generally, for $b \in \mathscr{L}$, let $(T(b), \hat{T}(b))=\sum_{i \leq j}\left(T\left(b_{i j}\right), \hat{T}\left(b_{i j}\right)\right)$ where $b_{i j} \in \mathscr{B}_{i j}$ are the components of $b$. One checks that $(T(e), \hat{T}(e))=\left(I, \frac{1}{2} I\right)$ 
and $T(b) e=b$. Thus

$$
\left(X_{0}(T(b), \hat{T}(b))\right)_{i e}=i b, \quad b \in \mathscr{V} .
$$

Now set

$$
\begin{aligned}
\mathfrak{g} & =\mathfrak{g}_{-1} \oplus \mathfrak{g}_{-1 / 2} \oplus \mathfrak{I}_{0} \\
\mathfrak{S}_{0} & =\{(T(b), \hat{T}(b)): b \in \mathscr{V}\} .
\end{aligned}
$$

We will show that $\mathfrak{g}_{0}$, and hence $\mathfrak{g}$, is a Lie subalgebra with $\mathfrak{g} \cap \mathfrak{E}=0$ and $\operatorname{dim} \mathfrak{g}=\operatorname{dim}_{\mathbf{R}} D$. Thus $S=\exp \mathfrak{S}$ acts simply-transitively on $D$ (cf. the argument in [1], Lemma 2.4). The diffeomorphism $S \ni s \rightarrow s(i e) \in D$ is equivariant with respect to the left $S$ actions so $S$ inherits a left-invariant Riemannian metric, determined by $\langle\rangle \mid$,$s , and a left-invariant complex$ structure determined by a map $j: \mathfrak{g} \rightarrow \mathfrak{g}$. Since $(j X)_{i e}^{*}=i\left(X_{i e}^{*}\right)$, we have

$$
\begin{aligned}
j\left(X_{-1}[b]\right)^{+} & =(T(b), \hat{T}(b)), & b & \in \mathscr{V} \\
j\left(X_{-1 / 2}[d]\right)^{+} & =\left(X_{-1 / 2}[i d]\right)^{+}, & & d \in \mathscr{U} .
\end{aligned}
$$

To demonstrate our claim, first note that $\mathscr{L}=\bigoplus_{1 \leq i \leq j \leq r} \mathscr{B}_{i j}$ defines an $r$-Peirce decomposition in the sense of [7, 1.3] and, by [12, Theorem 3.1], we even know that $\mathscr{L}=\bigoplus_{1 \leq m \leq n \leq q} \mathscr{L}_{m n}$ is a $(q-k)$-decomposition (relative to $\left.e_{11}, \ldots, e_{q q}\right)$. Define $I_{j} \subset\{1, \ldots, r\}$ by $e_{j j}=\sum_{k \in I_{j}} d_{k}$. Then

$$
\mathscr{L}_{m n}=\bigoplus_{\substack{i \in I_{m} \\ j \in I_{n}}} \mathscr{B}_{i j}
$$

and [7, Lemma 1.1.a] shows that $L_{d_{i}}\left(b_{i j}\right)=L_{e_{m}}\left(b_{i j}\right)$ if $i \in I_{m}, j \in I_{n}$, $I_{m} \cap I_{n}=\varnothing$ and $b_{i j} \in \mathscr{B}_{i j}$. We define the vector spaces $\mathfrak{g}_{m n}^{\mathscr{L}}, \mathfrak{g}^{\mathscr{L}}, \mathfrak{g}_{i j}^{\mathscr{B}}, \mathfrak{g}^{\mathscr{B}}$ as in [7, §3] but with reversed order of the subscripts, i.e. we consider the adjoints of endomorphisms of [7]. Let now $m<n$, then $i<j$ if $i \in I_{m}$ and $j \in I_{n}$ whence

$$
\mathfrak{g}_{m n}^{\mathscr{L}}=\bigoplus_{\substack{i \in I_{m} \\ j \in I_{n}}} \mathfrak{g}_{i j}^{\mathscr{B}} \quad \text { and } \quad \mathfrak{g}_{m n}^{\mathscr{L}} \subset \mathrm{g}^{\mathscr{D}} .
$$

This implies $\mathfrak{n}:=\bigoplus_{m<n} \mathfrak{g}_{m n}^{\mathscr{L}} \subset \mathfrak{g}^{\mathscr{B}}$. From the definition of the space $\mathfrak{g}_{m n}^{\mathscr{L}}$, it is clear that $L_{d_{i}}\left(b_{i j}\right) \in \mathrm{g}_{n n}^{\mathscr{L}}$ if $i, j \in I_{n}$. Hence $\mathrm{g}^{\mathscr{B}} \subset \mathrm{g}^{\mathscr{L}}$. By [7, Theorem 3.3], we know that $\mathrm{g}^{\mathscr{L}}$ is a Lie algebra with $\left[\mathrm{g}^{\mathscr{L}}, \mathfrak{n}\right] \subset \mathfrak{n}$ and $\left[\mathfrak{g}_{m m}^{\mathscr{L}}, \mathfrak{g}_{n n}^{\mathscr{L}}\right]=0$ if $m \neq n$. Now let $T_{1}, T_{2}$ be elements of $\mathrm{g}^{\mathscr{B}}$. To prove $\left[T_{1}, T_{2}\right]$ is in $\mathrm{g}^{\mathscr{L}}$, we may assume that each $T_{k}$ is in some $\mathrm{g}_{i j}^{\mathscr{B}}$. We also may assume $T_{1}, T_{2} \notin \mathfrak{n}$. Therefore $T_{1} \in \mathrm{g}_{m m}^{\mathscr{L}}, T_{2} \in \mathrm{g}_{n n}^{\mathscr{L}}$. If $m \neq n$, we get $\left[T_{1}, T_{2}\right]=0$, so we may assume $m=n$. It therefore suffices to prove that $\mathrm{g}^{\mathscr{B}} \cap \mathfrak{g}_{n n}^{\mathscr{L}}$ is a Lie algebra. From [7, Lemma 1.1], we know that $\mathrm{g}_{n n}^{\mathscr{L}}$ acts trivially on $\mathscr{L}_{l m}$ if $n \notin\{l, m\}$ and from [7, Lemmas 2.3 and 3.1], we find that the map $\left(L\left(b_{n n}\right) \mid \mathscr{L}_{n n}\right) \rightarrow\left(L\left(b_{n n}\right) \mid \mathscr{L}_{n p}\right), \quad n \neq p$, induces a homomorphism 
$\left(\mathrm{g}_{n n}^{\mathscr{L}} \mid \mathscr{L}_{n n}\right) \rightarrow$ End $\mathscr{L}_{n p}$ of Lie algebras. Hence, to see that $\mathrm{g}^{\mathscr{B}} \cap \mathrm{g}_{n n}^{\mathscr{L}}$ is a Lie algebra we only have to consider $\left(\mathrm{g}^{\mathscr{D}} \cap \mathrm{g}_{n n}^{\mathscr{L}}\right) \mid \mathscr{L}_{n n}$. But now we use the fact that $\left(\mathfrak{g}^{\mathscr{B}} \cap \mathfrak{g}_{n n}^{\mathscr{L}}\right) \mid \mathscr{L}_{n n}=\mathrm{g}^{\mathscr{C}}$, where $\mathscr{C}$ is the Peirce decomposition induced on $\mathscr{L}_{n n}$ from $\mathscr{B}$, and apply [10, Lemma 4.2 (a)] to conclude that $\mathrm{g}^{\mathscr{L}}$, and hence $\mathrm{g}^{\mathscr{B}}$, is a Lie algebra. Finally, we apply [12, Lemma 3.10] to see that $\mathfrak{g}_{0}$ is a Lie algebra.

We note from the definition of $L_{d_{j}}\left(x_{i j}\right), i<j$, that these endomorphisms are block matrices (in the obvious base) having zeroes in and below the diagonal. Therefore $\mathfrak{n}$ is a nilpotent Lie algebra. Moreover, $\left[\mathfrak{g}^{\mathscr{B}}, \mathrm{g}^{\mathscr{B}}\right] \subset \mathfrak{n}$ whence $\mathfrak{g}^{\mathscr{B}}$ is solvable and hence so is $\mathfrak{g}_{0}$. Finally, note that $\left(L(X), \frac{1}{2} \phi(X)\right), X \in \mathscr{X}$, has only real eigenvalues so $\mathfrak{S}_{0}$ is in fact split over R. By standard results of Koszul [25] (cf. discussion in [28], pp. 47-48), this shows that $(\mathfrak{g}, j)$ is a normal $j$-algebra with admissible form $\omega$ determining the Bergman metric. We also deduce that the product $a \Delta b=$ $T(a) b$ is the Vinberg (clan) algebra on $\mathscr{V}$.

Let $a$ denote the abelian subalgebra of $\mathfrak{s}$ given by

$$
\mathfrak{a}=\bigoplus_{k=1}^{r} \mathbf{R}\left(T\left(d_{k}\right), \hat{T}\left(d_{k}\right)\right)
$$

Define linear functionals $\varepsilon_{k}$ on a by

$$
\varepsilon_{k}\left(j X_{l}\right)=\delta_{k l} \text { where } X_{l}=\left(X_{-1}\left[d_{l}\right]\right)^{+} .
$$

For each linear functional $\alpha$ on $\mathfrak{a}$, let $\mathfrak{n}_{\alpha}$ be the root space of the adjoint action of $\mathfrak{a}$ on $\mathfrak{g}$. Then $\mathfrak{g}$ is the orthogonal direct sum of $\mathfrak{n}_{0}=\mathfrak{a}$ and the root spaces (some of which may be null)

$$
\begin{aligned}
& \mathfrak{n}_{\varepsilon_{k}}=\mathbf{R} X_{k} \\
& \mathfrak{n}_{\varepsilon_{k} / 2}=\left\{X_{-1 / 2}\left[\phi\left(d_{k}\right) u\right]^{+}: u \in \mathscr{U}\right\} \quad \text { for } 1 \leq k \leq r \text {, } \\
& \mathfrak{n}_{\left(\varepsilon_{k}-\varepsilon_{l}\right) / 2}=\left\{\left(T\left(b_{k l}\right), \hat{T}\left(b_{k l}\right)\right): b_{k l} \in \mathscr{B}_{k l}\right\} \\
& \mathfrak{n}_{\left(\varepsilon_{k}+\varepsilon_{l}\right) / 2}=\left\{X_{-1}\left[b_{k l}\right]^{+}: b_{k l} \in \mathscr{B}_{k l}\right\}
\end{aligned}
$$

One sees that $\mathfrak{n}=[\mathfrak{Z}, \mathfrak{Z}]$ and that $\mathfrak{n}$ is the orthogonal complement of $\mathfrak{a}$. Further, with our normalization, we have $\omega\left(X_{k}\right)=\frac{1}{4} \sigma\left(d_{k}, d_{k}\right)$ and $\sigma(e, e)$ $=\operatorname{dim}_{\mathbf{R}}\left(\mathscr{V}^{\mathbf{C}}\right)+\operatorname{dim}_{\mathbf{C}} \mathscr{U}$ (compare [3, Th. 4] which uses a different normalization, also, the last formula is given incorrectly in [11, 12]).

Now we want to relate the above constructions to the work of Azencott and Wilson. Following $[12 ; \S 7 ., 3]$, let $\mathscr{V}^{\prime}=\mathscr{P}_{1}, \mathscr{U}=\mathscr{P}_{1 / 2}$, $F^{\prime}=F \mid \mathscr{U}^{\prime} \times \mathscr{U}^{\prime}, \Omega^{\prime}$ equal the projection of $\Omega$ onto $\mathscr{V}^{\prime}$ (with respect to $\sigma$ ) and $D^{\prime}=\left\{(Z, U) \in\left(\mathscr{V}^{\prime}\right)^{\mathbf{C}} \times \mathscr{U}^{\prime}: \operatorname{Im}: Z-F^{\prime}(U, U) \in \Omega^{\prime}\right\}$. Then $D^{\prime}$ is a 
symmetric Siegel domain and the algebra defined thereby on $\mathscr{V}^{\prime}$ agrees with the subalgebra structure of $\mathscr{P}_{1}$ from $\mathscr{L}$. In general, the superscript ' will denote objects defined by $D^{\prime}$. e.g. $e^{\prime}$ will be the identity of $\mathscr{L}^{\prime}=\mathscr{P}_{1}$ and $g^{\prime}$ will be the Lie algebra of $\operatorname{Aut}\left(D^{\prime}\right)$. Note that on each simple factor of $D^{\prime}, \sigma$ is a multiple of $\sigma^{\prime}$. There is a graded subalgebra $g^{\prime \prime}$ of $g$ so that the map $X^{*} \rightarrow X^{*} \mid D^{\prime}$ (i.e., first $X^{*}$ is extended to a vector field on $\mathscr{V}^{\mathbf{C}} \times \mathscr{U}$ and then restricted to $\left.D^{\prime}\right)$ is a Lie isomorphism onto $\left(g^{\prime}\right)^{*}$. More precisely, $\mathfrak{g}^{\prime \prime}=\mathfrak{g}_{-1}^{\prime \prime} \oplus \mathfrak{g}_{-1 / 2}^{\prime \prime} \oplus \mathfrak{g}_{0}^{\prime \prime} \oplus \mathfrak{g}_{1}^{\prime \prime}$ where

$$
\begin{gathered}
\mathfrak{g}_{-1}^{\prime \prime}=\left\{X_{-1}[a]: a \in \mathscr{P}_{1}\right\}^{+}, \quad \mathfrak{g}_{-1 / 2}^{\prime \prime}=\left\{X_{-1 / 2}[d]: d \in \mathscr{P}_{1 / 2}\right\}^{+}, \\
\mathfrak{g}_{1 / 2}^{\prime \prime}=\mathfrak{g}_{1 / 2}, \quad \mathfrak{g}_{1}^{\prime \prime}=\mathfrak{g}_{1},
\end{gathered}
$$

and $\mathfrak{g}_{0}^{\prime \prime}$ is a subalgebra of $\mathfrak{g}_{0}$.

$D^{\prime}$ is not contained in $D$, however, letting $e_{0}=e-e^{\prime}$ one has $\Omega^{\prime}+e_{0} \subset \Omega$ so $D^{\prime \prime}=D^{\prime}+i e_{0}$ is an equivalent submanifold of $D$. One knows $T\left(e_{0}\right)=0$ for $(T, \hat{T}) \in \mathfrak{g}_{0}^{\prime \prime}$ and $\phi\left(e_{0}\right) d=0$ for $d \in \mathscr{P}_{1 / 2}$. Using this one proves

$$
\left.X^{*}\right|_{(Z, U)}=\left.X^{*}\right|_{\left(Z+i e_{0}, U\right)} \quad \text { for }(Z, U) \in D^{\prime}, X \in \mathrm{g}^{\prime \prime},
$$

which shows how the isomorphism $\left(\mathfrak{g}^{\prime \prime}\right)^{*} \simeq\left(\mathfrak{g}^{\prime}\right)^{*}$ relates the various structures. For example, the Cartan involution on $\mathrm{g}^{\prime}$ relative to the symmetry at $i e^{\prime}$ is known from [11]. Pulling this back to $\mathfrak{g}^{\prime \prime}\left(\right.$ or $\left.\left(\mathfrak{g}^{\prime \prime}\right)^{*}\right)$ gives a Cartain involution $\theta$ defined by

$$
\begin{array}{ll}
\theta\left(X_{\lambda}[a]\right)=X_{-\lambda}[a] & \text { for } \lambda= \pm \frac{1}{2}, \pm 1, a \in \mathscr{P}_{1} \text { or } \mathscr{P}_{1 / 2} \\
\theta(T, \hat{T})=-\left(T^{t}, \hat{T}^{t}\right) & \text { for }(T, \hat{T}) \in \mathfrak{g}_{0}^{\prime \prime}
\end{array}
$$

where the superscript $t$ denotes adjoint with respect to $\sigma$ and $\rho$. The compact part of the corresponding Cartan decomposition is just the isotropy subalgebra $\mathfrak{f} \cap \mathrm{g}^{\prime \prime}$ for $D^{\prime \prime}$. This is meaningful since we also see that $\exp \mathfrak{g}^{\prime \prime}$ leaves $D^{\prime \prime}$ invariant and is transitive. [18, p. 178] then implies that the metric induced on $D^{\prime \prime}$ from $(D, g)$ is symmetric and hence on each irreducible component of $D^{\prime \prime}$ is a multiple of its Bergman metric.

Now let $q$ be the sum of the semi-simple parts of the structure algebras of $\mathscr{L}_{11}, \mathscr{L}_{22}, \ldots, \mathscr{L}_{q q}^{0}$ and let $\mathrm{q}_{U}$ be the semi-simple part of the compact algebra $\mathrm{t}_{a}$ (note $\mathrm{t}_{a} \subset \mathfrak{f}_{0}$; see $[12, \S 7.2]$ for definition). The elements of $q$ can be canonically extended so that $q \oplus a_{U}$ becomes a subalgebra of $\mathfrak{g}_{0}$. Let $\mathfrak{r}$ be the radical of $\mathfrak{g}$ which is a graded ideal of the form

$$
\mathfrak{r}=\mathfrak{r}_{-1} \oplus \mathfrak{r}_{-1 / 2} \oplus \mathfrak{r}_{0}
$$


where $\mathfrak{g}_{-1}=\mathfrak{r}_{-1} \oplus \mathfrak{g}_{-1}^{\prime \prime}, \mathfrak{g}_{-1 / 2}=\mathfrak{r}_{-1 / 2} \oplus \mathfrak{g}_{-1 / 2}^{\prime \prime}$ (orthogonal with respect to $\langle\rangle$,

(20)

$$
\begin{aligned}
& \mathfrak{g}_{0}=\mathfrak{r}_{0} \oplus \mathfrak{g}_{0}^{\prime \prime} \oplus \mathfrak{q} \oplus \mathfrak{q}_{U} \\
& \mathfrak{r}_{0}=\mathfrak{n}^{\phi} \oplus L\left(\text { center }\left(\mathscr{X} \ominus \mathscr{P}_{1}\right)\right) \oplus \text { center } \mathrm{t}_{a} \\
& \mathfrak{n}^{\phi}=\bigoplus_{1 \leq i \leq j \leq q}\left\{\left(L_{e_{j j}}\left(Y_{i j}\right), \frac{1}{2} \phi\left(Y_{i j}\right) \phi\left(e_{i i}\right)\right): Y_{i j} \in \mathscr{L}_{i j}\right\},
\end{aligned}
$$

$\operatorname{dim} \mathfrak{n}^{\phi}=\operatorname{dim} \mathscr{L}-\operatorname{dim} \mathscr{X}$.

In addition (cf. (7)), $\mathfrak{f}_{0}=\left(\mathfrak{f}_{0} \cap \mathfrak{g}^{\prime \prime}\right) \oplus \sum \mathfrak{f}_{0 j} \oplus \mathfrak{t}_{a}$ where $\mathfrak{f}_{0 j}=$ $\operatorname{span}\left\{\left[L\left(X_{j j}\right), L\left(Y_{j j}\right)\right]: X_{j j}, Y_{j j} \in \mathscr{L}_{j j}\right\}$ is isomorphic to Der $\mathscr{L}_{j j}$ (clearly, the center of $\mathfrak{I}_{0}$ is the sum of the centers of the summands of $\mathfrak{f}_{0}$; we note center $\left.\left(\mathfrak{t}_{a}\right)=\mathfrak{f}_{0} \cap \mathfrak{r}_{0}\right)$.

We now define

$$
\begin{aligned}
& \mathfrak{g}_{01}=\left\{(T(b), \hat{T}(b)): b \in \mathscr{P}_{1}\right\}=j\left(\mathfrak{g}_{-1}^{\prime \prime}\right) \\
& \mathfrak{S}_{02}= \operatorname{span}\left\{\left\{\left(T\left(b_{i j}\right), \hat{T}\left(b_{i j}\right)\right): i<j, b_{i j} \in \mathscr{B}_{i j} \subset \mathscr{X} \ominus \mathscr{P}_{1}\right\}\right. \\
& \cup\left\{(T(b), \hat{T}(b)): b=\sum b_{i i}, b_{i i} \in \mathscr{B}_{i l} \subset \mathscr{X} \ominus \mathscr{P}_{1}\right.\left.\left.\quad \text { and } \sigma\left(b, \operatorname{center}\left(\mathscr{X} \ominus \mathscr{P}_{1}\right)\right)=0\right\}\right\} \\
& \mathfrak{g}^{\prime \prime}=\mathfrak{g}_{-1}^{\prime \prime} \oplus \mathfrak{g}_{-1 / 2}^{\prime \prime} \oplus s_{01} \\
& \mathfrak{I}_{s s}=\mathfrak{g}^{\prime \prime} \oplus \mathfrak{g}_{02} \\
& \mathfrak{S}_{r}=\mathfrak{r}_{-1} \oplus \mathfrak{r}_{-1 / 2} \oplus \mathfrak{n}^{\phi} \oplus L\left(\operatorname{center}\left(\mathscr{X} \ominus \mathscr{P}_{1}\right)\right) \\
& \mathfrak{h}_{s s}=\mathfrak{g}^{\prime \prime} \oplus \mathfrak{q} .
\end{aligned}
$$

By [12, Theorem 7.8], $\mathfrak{g}=\left(\mathfrak{h}_{s s} \oplus \mathfrak{q}_{U}\right) \oplus \mathfrak{r}$ is a Levi decomposition of $\mathfrak{g}$, $\mathfrak{h}_{s s}$ is the sum of the noncompact ideals of the semi-simple component, and $\mathfrak{h}_{s s}=\mathfrak{g}^{\prime \prime} \oplus \mathfrak{q}$ is a direct sum of ideals. One checks that

$$
\begin{aligned}
& \mathfrak{I}^{\prime \prime} \text { is a subalgebra of } \mathfrak{g}^{\prime \prime} \text { with } \mathfrak{g}^{\prime \prime}=\left(\mathfrak{f} \cap \mathfrak{g}^{\prime \prime}\right) \oplus \mathfrak{g}^{\prime \prime} \\
& \mathfrak{S}_{02} \text { is a subalgera of } \mathfrak{q} \text { with } \mathfrak{q}=(\mathfrak{f} \cap \mathfrak{q}) \oplus \mathfrak{S}_{02}
\end{aligned}
$$

$\mathfrak{g}$ is the orthogonal direct sum $\mathfrak{S}_{s s} \oplus \mathfrak{S}_{r}$ of the subalgebra $\mathfrak{S}_{s s}=\mathfrak{g} \cap \mathfrak{h}_{s s}$ and the ideal $\mathfrak{g}_{r}=\mathfrak{g} \cap \mathfrak{r}$.

Now a Cartan involution $\boldsymbol{\theta}$ on $\mathfrak{h}_{s s}$ is given by (19) and

$$
\theta(T, \hat{T})=-\left(T^{t}, \hat{T}^{t}\right) \quad \text { for }(T, \hat{T}) \in q
$$


By standard results about structure algebras of formally real Jordan algebras, $\mathfrak{f} \cap q$ is the fixed point algebra of $\theta \mid q$. As before, [18, p. 178] shows that the orbit $\exp q(i e)=\exp s_{02}(i e)$ is Riemannian locally symmetric with respect to the induced metric. Further, (23) and the standard covariant derivative formula

$$
\begin{array}{r}
2\left\langle\nabla_{X} Y, Z\right\rangle=\langle[X, Y], Z\rangle+\langle[Z, X], Y\rangle+\langle[Z, Y], X\rangle, \\
X, Y, Z \in \mathfrak{g}
\end{array}
$$

show that the orbit $\exp \mathfrak{S}_{s s}(i e)=\exp \mathfrak{h}_{s s}(i e)$ is totally geodesic in $(D, g)$ and then $\exp \mathfrak{g}^{\prime \prime}(i e)$ and $\exp q(i e)$ are also seen to be totally geodesic.

Let

$$
\mathfrak{n}_{s s}=\mathfrak{n} \cap \mathfrak{S}_{s s}, \quad \mathfrak{n}_{r}=\mathfrak{n} \cap \mathfrak{I}_{r}
$$

and observe that (23) implies

$$
\mathfrak{n}=\mathfrak{n}_{s s} \oplus \mathfrak{n}_{r}, \quad\left[\mathfrak{g}_{s s}, \mathfrak{g}_{s s}\right]=\mathfrak{n}_{s s}
$$

Similarly, let

$$
\mathfrak{a}_{s s}=\mathfrak{a} \cap \mathfrak{g}_{s s}, \quad \mathfrak{a}_{r}=\mathfrak{a} \cap \mathfrak{g}_{r}
$$

and observe that

$$
\mathfrak{a}=\mathfrak{a}_{s s} \oplus \mathfrak{a}_{r}, \quad \mathfrak{g}_{s s}=\mathfrak{a}_{s s} \oplus \mathfrak{n}_{s s}, \quad \mathfrak{g}_{r}=\mathfrak{a}_{r} \oplus \mathfrak{n}_{r}
$$

follow easily from $\mathfrak{a}=\mathfrak{n}^{\perp}$, (23) and (27). We may order the idempotents $\left\{d_{i}\right\}$ so that $\left\{d_{1}, \ldots, d_{s}\right\} \subset \mathscr{X} \ominus \mathscr{P}_{1},\left\{d_{s+1}, \ldots, d_{r}\right\} \subset \mathscr{P}_{1}$ and further $\{1, \ldots, s\}$ is the disjoint union $\bigcup I_{j}^{\prime}$ where each $I_{j}^{\prime}$ is a set of consecutive indices and $\sum_{k \in I_{j}^{\prime}} d_{k}=c_{j}$ is the identity of a simple factor of $\mathscr{X} \ominus \mathscr{P}_{1}$. Then center $\left(\mathscr{X} \ominus \mathscr{P}_{1}\right)=\operatorname{span}\left\{c_{j}\right\}$ and $a_{r}=\oplus \mathbf{R}\left(T\left(c_{j}\right), \hat{T}\left(c_{j}\right)\right)$. Also if $i<j$ and $\mathscr{B}_{i j} \subset \mathscr{X} \ominus \mathscr{P}_{1}$ then $\mathscr{B}_{i j}$ is actually in one of these simple factors. These observations imply

$$
\mathfrak{n}_{s s} \text { is the sum of the root spaces } \mathfrak{n}_{\alpha}, \alpha \neq 0, \alpha \mid \mathfrak{a}_{r}=0 \text {. }
$$

Now for any $Y \in \mathfrak{n}_{s s},(25)$ and (30) imply that there exists $H \in \mathfrak{a}_{s s}$ so that the curvature $R(H, Y) H \neq 0$. It follows that $\exp a_{s s}(i e)$ is a maximal flat totally geodesic subspace of the Riemannian locally symmetric space $\exp \mathfrak{h}_{s s}(i e)$ which thus has rank equal to $\operatorname{dim} \mathfrak{a}_{s s}$. Let $\mathfrak{f}_{s s} \oplus \mathfrak{p}_{s s}, \mathfrak{f}_{s s}=$ $\mathfrak{f} \cap \mathfrak{h}_{s s}$, be the Cartan decomposition of $\mathfrak{h}_{s s}$ with respect to $\theta$. Since multiplicaiton in $\mathscr{L}$ is a symmetric operator with respect to $\sigma$, one gets that $\theta \mid \mathfrak{a}_{s s}=-I$. Thus $\mathfrak{a}_{s s} \subset \mathfrak{p}_{s s}$ and is maximal abelian. From (30) and the form of the roots $\alpha$, it is clear that we can order a basis of $\mathfrak{a}_{s s}$ so that $\mathfrak{n}_{s s}$ is contained in the sum of the positive root spaces of the $\mathfrak{a}_{s s}$ action on $\mathfrak{h}_{s s}$. Since $\mathfrak{h}_{s s}=\mathfrak{f}_{s s} \oplus \mathfrak{S}_{s s}$, it follows that

$$
\mathfrak{Z}_{s s} \text { is an Iwasawa subalgebra of } \mathfrak{h}_{s s} \text {. }
$$


Note also that (26)-(29) imply that for any nonzero $H \in \mathfrak{a}_{s s}, \operatorname{ad}_{\mathfrak{n}_{s s}} H$ is not identically 0 .

For a later theorem, we require the following technical lemma.

LEMMA. $\left(\operatorname{ad}_{\mathfrak{\Xi}_{r}} X\right)^{t}=-\operatorname{ad}_{\mathfrak{\vartheta}_{r}} \theta X$ for $X \in \mathfrak{h}_{s s}$.

Proof. The computation will be done in $\mathrm{g}^{*}$ with $\theta$ and $\langle$,$\rangle pulled$ back to $\mathfrak{h}_{s s}^{*}$ and $\mathfrak{S}^{*}$, respectively.

If $X \in \mathfrak{h}_{s s} \cap \mathfrak{g}_{-1}$, then $X^{*}=X_{-1}[a]$ for $a \in \mathscr{P}_{1}$ and $\theta X^{*}=$ $X_{1}[a] \in \mathfrak{g}_{1}^{*}$. Since $\left[\mathfrak{g}_{1},\left(\mathfrak{g}_{r}\right)_{-1 / 2}+\left(\mathfrak{g}_{r}\right)_{0}\right] \subset\left(g_{1 / 2} \oplus \mathfrak{g}_{1}\right) \cap \mathfrak{r}=0$ and since the graded components of $\mathfrak{s}$ are orthogonal, it suffices to prove

$$
\left\langle\left[X_{1}[a], X_{-1}[b]\right], X_{0}(T, \hat{T})\right\rangle=-\left\langle X_{-1}[b],\left[X_{-1}[a], X_{0}(T, \hat{T})\right]\right\rangle
$$

where $X_{-1}[b]$ is a typical element of $r_{-1}^{*}\left(\right.$ so $\left.\sigma\left(b, \mathscr{P}_{1}\right)=0\right)$ and $X_{0}(T, \hat{T})$ is a typical element of $\left(\mathfrak{S}_{r}\right)_{0}^{*}$. But

$$
\begin{gathered}
\left\langle\left[X_{1}[a], X_{-1}[b]\right], X_{0}(T, \hat{T})\right\rangle \\
=-\left\langle X_{0}\left(2 L_{a}(b), \phi(b) \phi(a)\right), X_{0}(T, \hat{T})\right\rangle \\
=-g_{i e}\left(2 L_{a}(b)(i e), T(i e)\right)=-\frac{1}{2} \sigma(a b, T e), \\
\left\langle X_{-1}[b],\left[X_{-1}[a], X_{0}(T, \hat{T})\right]\right\rangle=\left\langle X_{-1}[b], X_{-1}[T a]\right\rangle=\frac{1}{4} \sigma(b, T a) .
\end{gathered}
$$

Now $T^{t}=2 L(T e)-T\left[7\right.$, p. 88] and $T^{t} \mathscr{P}_{1}=0$ (it suffices to assume $T \in \mathfrak{n}$, whence $T=L e_{j}\left(b_{i j}\right), i<j$, and use $L_{e_{1}}\left(\mathscr{P}_{1}\right)=0$ by [7, Lemma $1.1 \mathrm{a}, \mathrm{p}$. 82] $)$ so $\sigma(b, T a)=\sigma\left(T^{t} b, a\right)=2 \sigma(L(T e) b, a)=2 \sigma(a b, T e)$, proving this case.

If $X \in \mathfrak{h}_{s s} \cap g_{-1 / 2}$, then $X^{*}=X_{-1 / 2}[d]$ for $d \in \mathscr{P}_{1 / 2}$ and $\theta X^{*}=$ $X_{1 / 2}[d] \in g_{1 / 2}^{*}$. Arguing as before, it suffices to prove

$$
\begin{aligned}
& \left\langle\left[X_{1 / 2}[d], X_{-1}[b]+X_{-1 / 2}[w]\right], X_{-1 / 2}\left[w^{\prime}\right]+X_{0}(T, \hat{T})\right\rangle \\
& \quad=\left\langle X_{-1}[b]+X_{1 / 2}[w],\left[X_{-1 / 2}[d], X_{-1 / 2}\left[w^{\prime}\right]+X_{0}(T, \hat{T})\right\rangle\right.
\end{aligned}
$$

for $b \in \mathscr{P}_{1}^{\perp}, w, w^{\prime} \in \mathscr{P}_{1 / 2}^{\perp},(T, \hat{T}) \in\left(\mathfrak{S}_{r}\right)_{0}^{*}$. But

$$
\begin{gathered}
\left\langle\left[X_{1 / 2}[d], X_{-1}[b]+X_{-1 / 2}[w]\right], X_{-1 / 2}\left[w^{\prime}\right]+X_{0}(T, \hat{T})\right\rangle \\
=\left\langle-X_{-1 / 2}[i \phi(b) d]-2(F(w, \phi(\bar{Z}) d)+F(\phi(Z) d, w),\right. \\
-\phi(F(U, w)) d+\phi(F(w, d)) U+\phi(F(U, d)) w), \\
\left.X_{-1 / 2}\left[w^{\prime}\right]+X_{0}(T, \hat{T})\right\rangle \\
=g_{i e}\left(-i \phi(b) d-2(2 i \operatorname{Re} F(w, d)), w^{\prime}+i T e\right) \\
=\operatorname{Re}\left(-\sigma(\operatorname{Re} F(w, d), T e)-\rho\left(i \phi(b) d, w^{\prime}\right)\right)
\end{gathered}
$$


while

$$
\begin{aligned}
\left\langle X_{-1}\right. & {\left.[b]+X_{1 / 2}[w],\left[X_{-1 / 2}[d], X_{-1 / 2}\left[w^{\prime}\right]+X_{0}(T, \hat{T})\right]\right\rangle } \\
& =\left\langle X_{-1}[b]+X_{1 / 2}[w], X_{-1}\left[-4 \operatorname{Im} F\left(d, w^{\prime}\right)\right]+X_{-1 / 2}[\hat{T} d]\right\rangle \\
& =g_{i e}\left(b+w,-4 \operatorname{Im} F\left(d, w^{\prime}\right)+\hat{T} d\right) \\
& =\operatorname{Re}\left(-\sigma\left(b, \operatorname{Im} F\left(d, w^{\prime}\right)\right)+\rho(w, \hat{T} d)\right) .
\end{aligned}
$$

Now

$-\operatorname{Re} \rho\left(i \phi(b) d, w^{\prime}\right)$

$$
\begin{aligned}
& =\operatorname{Im} \rho\left(\phi(b) d, w^{\prime}\right)=\frac{1}{2} \operatorname{Im}\left(\rho\left(\phi(b) d, w^{\prime}\right)-\rho\left(\phi(b) w^{\prime}, d\right)\right) \\
& =\operatorname{Re}(1 / 2 i) \sigma\left(b, F\left(d, w^{\prime}\right)-F\left(w^{\prime}, d\right)\right)=\operatorname{Re} \sigma\left(b, \operatorname{Im} F\left(d, w^{\prime}\right)\right) .
\end{aligned}
$$

Also

$$
\begin{aligned}
-\operatorname{Re} \sigma & (\operatorname{Re} F(w, d), T e)=-\operatorname{Re} \sigma(F(w, d), T e) \\
& =-\operatorname{Re} \sigma\left(F(w, d), T^{*} e\right)=-\operatorname{Re} \sigma(T F(w, d), e) \\
& =-\operatorname{Re} \sigma(F(\hat{T} w, d)+F(w, \hat{T} d), e) \\
& =-\operatorname{Re} \rho(\hat{T} w, d)-\operatorname{Re} \rho(w, \hat{T} d)
\end{aligned}
$$

and note that $\rho(\hat{T} w, d)=0$ since $\hat{T}^{*} \mid \mathscr{P}_{1 / 2}=0$. (This follows from the form of $(T, \hat{T}) \in\left(\mathfrak{S}_{r}\right)_{0}^{*}$ and $\phi\left(X_{q q}\right) \phi\left(e_{j j}\right) U=0$ if $j \neq q[12 ; \S 3]$.)

Finally, if $X \in \mathfrak{h}_{s s} \cap \mathfrak{g}_{0}$, the $X^{*}=X_{0}(T, \hat{T})$ and $\theta X^{*}=-X_{0}\left(T^{t}, \hat{T}^{t}\right)$. Then

$$
\begin{gathered}
\left\langle\left[-X_{0}\left(T^{t}, \hat{T}^{t}\right), X_{-1}[a]+X_{-1 / 2}[d]+X_{0}(R, \hat{R})\right]\right. \\
\left.X_{-1}\left[a^{\prime}\right]+X_{-1 / 2}\left[d^{\prime}\right]+X_{0}\left(R^{\prime}, \hat{R}^{\prime}\right)\right\rangle \\
=\left\langle X_{-1}\left[T^{t} a\right]+X_{-1 / 2}\left[\hat{T}^{t} d\right]+X_{0}\left(\left[T^{t}, R\right],\left[\hat{T}^{t}, \hat{R}\right]\right)\right. \\
\left.X_{-1}\left[a^{\prime}\right]+X_{-1 / 2}\left[d^{\prime}\right]+X_{0}\left(R^{\prime}, \hat{R}^{\prime}\right)\right\rangle \\
=\frac{1}{4} \sigma\left(T^{t} a, a^{\prime}\right)+\rho\left(\hat{T}^{t} d, d^{\prime}\right)+\frac{1}{4} \sigma\left(\left[T^{t}, R\right] e, R^{\prime} e\right)
\end{gathered}
$$

while

$$
\begin{gathered}
\left\langle X_{-1}[a]+X_{-1 / 2}[d]+X_{0}(R, \hat{R}),\right. \\
\left.\left[X_{0}(T, \hat{T}), X_{-1}\left[a^{\prime}\right]+X_{-1 / 2}\left[d^{\prime}\right]+X_{0}\left(R^{\prime}, \hat{R}^{\prime}\right)\right]\right\rangle \\
=-\frac{1}{4} \sigma\left(a, T a^{\prime}\right)-\rho\left(d, \hat{T} d^{\prime}\right)-\frac{1}{4} \sigma\left(\operatorname{Re},\left[T, R^{\prime}\right] e\right) .
\end{gathered}
$$

Now as before, one has $U^{t}=2 L(U e)-U$ for any $(U, \hat{U}) \in g_{0}$; in particular, $U e=U^{t} e$. Thus

$$
\begin{aligned}
\sigma\left(\left[R, R^{\prime}\right] e, T e\right) & =\sigma\left(\left[R, R^{\prime}\right]^{t} e, T e\right)=\sigma\left(\left(R^{\prime}\right)^{t} R^{t} e-R^{t}\left(R^{\prime}\right)^{t} e, T e\right) \\
& =\sigma\left(\operatorname{Re}, R^{\prime} T e\right)-\sigma\left(R^{\prime} e, R T e\right) .
\end{aligned}
$$


By assumption, $(R, \hat{R}), \quad\left(R^{\prime}, \hat{R}^{\prime}\right) \in \mathfrak{n}^{\phi} \oplus L\left(\operatorname{center}\left(\mathscr{X} \ominus \mathscr{P}_{1}\right)\right)$ so $\left(\left[R, R^{\prime}\right],\left[\hat{R}, \hat{R}^{\prime}\right]\right) \in \mathfrak{n}^{\phi}$. Now

$$
T e \in \mathscr{P}_{1} \oplus\left\{b \in \mathscr{X} \ominus \mathscr{P}_{1}: \sigma\left(b, \operatorname{center}\left(\mathscr{X} \ominus \mathscr{P}_{1}\right)\right)=0\right\}
$$

while from the definition of $\mathfrak{n}^{\phi},\left[R, R^{\prime}\right] e$ is orthogonal to $\mathscr{X}$. Thus (34) vanishes. Using this, $T e=T^{t} e$, and expanding the terms in (32) and (33) involving $R, R^{\prime}, T$, one shows (32) $=-(33)$, as required.

REMARK. In a forthcoming paper we will include the proof that $g$ is a complete isometry algebra in the sense of [2].

2. Isotropy representations. Suppose $G$ is the identity component of the isometry group of a Riemannian space $(D, g), K$ is the isotropy subgroup at a point $b \in D$, and $S$ is a Lie subgroup of $G$ acting simply transitively on $D$. Let $\mathfrak{g}, \mathfrak{f}, \mathfrak{g}$ be the corresponding Lie algebras and $\langle$, the inner product on $\&$ corresponding to the Riemannian metric via

$$
\left.\mathfrak{g} \ni X \rightarrow X^{*}\right|_{b} \in T_{b} D \text {. }
$$

Let

$$
\begin{array}{ll}
\Lambda: K \rightarrow \mathcal{O}\left(T_{b} D, g_{b}\right), & \Lambda(k)=\left.k_{*}\right|_{b} \\
\lambda: k \rightarrow \mathfrak{S} \mathfrak{O}\left(T_{b} D, g_{b}\right), & \lambda(X)=\left.\frac{d}{d t}\right|_{t=0} \Lambda(\exp t X)=-\left.\operatorname{ad} X^{*}\right|_{b}
\end{array}
$$

be the isotropy representations. Here ad $\left.X^{*}\right|_{b}$ means that for any $V \in T_{b} D$ and any vector field $\tilde{V}$ extending $V$, we have

$$
\lambda(X) V=-\left[X^{*}, \tilde{V}\right]_{b}=\nabla_{V} X^{*} .
$$

We consider $\Lambda$ and $\lambda$ as representations on $\mathfrak{s}$ via (35). Properties of $\Lambda$ and $\lambda$ are detailed in $[2, \S 2]$. In fact, the following theorem is implicit in the work of Azencott-Wilson; we have extracted from [2] just what is needed for the proof. A similar theorem is explicitly given in the thesis of H. L. Williams [32]. Since we are only concerned with the conclusions about the isotropy representation our formulation can be more general.

THEOREM 1. With the notation above, suppose there are subalgebras $\mathfrak{S}_{1}$, $\mathfrak{u}_{1}, \mathfrak{u}, \mathfrak{g}_{2}$ of $\mathrm{g}$ such that

(1.1) $\mathrm{g}$ is the vector space direct sum $\mathfrak{I}_{1} \oplus \mathfrak{u}_{1} \oplus \mathfrak{u} \oplus \mathfrak{I}_{2}$.

(1.2) $\mathfrak{g}_{2}$ is an ideal and $\left[\mathfrak{u}, \mathfrak{g}_{1}\right]=0$.

(1.3) $\mathfrak{f}=\mathfrak{H}_{1} \oplus \mathfrak{H}$ is the isotropy subalgebra at $b$.

(1.4) $\mathfrak{h}_{1}=\mathfrak{g}_{1} \oplus \mathfrak{u}_{1}$ is a semi-simple subalgebra with no compact ideals and $\mathfrak{I}_{1} \oplus \mathfrak{u}_{1}$ is an Iwasawa decomposition with solvable factor $\mathfrak{g}_{1}$ and maximal compact factor $\mathfrak{H}_{1}$.

(1.5) Letting $\mathfrak{g}=\mathfrak{g}_{1} \oplus \mathfrak{g}_{2}$ and $S$ the corresponding analytic subgroup of $G, S$ acts simply transitively on $D$. 
Let $\boldsymbol{\theta}$ be the Cartan involution with Cartan decomposition $\mathfrak{h}_{1}=$ $\mathfrak{u}_{1} \oplus \mathfrak{p}_{1}$. Let $P$, resp. $Q$, be projections of $\mathfrak{h}_{1}$ onto $\mathfrak{p}_{1}$, resp. $\mathfrak{u}_{1}$, so $P=$ $\frac{1}{2}(I-\theta), Q=\frac{1}{2}(I+\theta)$. Let $\beta$ be a bilinear form on $\mathfrak{h}_{1}$ which is the (orthogonal) sum of multiples of the Killing form on each simple ideal.

We further assume that

(1.6) The inner product $\langle$,$\rangle obtained from the Riemannian metric$ via (35) satisfies

$(1.6 .1)\left\langle\mathfrak{g}_{1}, \mathfrak{g}_{2}\right\rangle=0$

(1.6.2) $\langle X, Y\rangle=\beta(P X, P Y)$ for $X, Y \in \mathfrak{g}_{1}$

(1.6.3) $\left(\operatorname{ad} \mathfrak{g}_{2} X\right)^{t}=-\operatorname{ad}_{\mathfrak{g}_{2}} \theta X$ for $X \in \mathfrak{g}_{1}$, where transpose is with respect to $\langle$,$\rangle .$

Let $\mathfrak{m}_{1}=\left\{X \in \mathfrak{u}_{1}:\right.$ ad $\left.X\left(\mathfrak{g}_{1}\right) \subset \mathfrak{g}_{1}\right\}$ and $l_{1}=\left\{X \in \mathfrak{u}_{1}: \beta\left(X, \mathfrak{m}_{1}\right)=\right.$ $0\}$. Then

(1.7) $\lambda(X)=\operatorname{ad}_{\mathfrak{z}} X$ for $X \in \mathfrak{m}_{1} \oplus \mathfrak{u}$ and $\lambda\left(\mathfrak{m}_{1} \oplus \mathfrak{u}\right)$ is the algebra of skew-symmetric derivations of $(\mathfrak{g},\langle\rangle$,

(1.8) $\lambda\left(l_{1}\right)=\left\{\nabla_{Y}: Y \in \mathfrak{n}_{1}=\left[\mathfrak{g}_{1}, \mathfrak{g}_{1}\right]\right\}$.

REMARK. (a) Condition (1.6.2) just says that the induced metric on $\exp \mathfrak{g}_{1} \cdot b$ is Riemannian locally symmetric.

(b) One easily derives for $X \in \mathfrak{f}$ a unique representation $\lambda(X)=\nabla_{a}$ $+\operatorname{ad} b, a \in \mathfrak{n}_{1}, b \in \mathfrak{m}_{1}+\mathfrak{u}$.

(c) As $\mathfrak{h}_{1}$ contains no compact ideals it is easy to see that $\mathfrak{u}_{1}$ is gemerated by $l_{1}$.

Proof. Clearly $\mathfrak{m}_{1} \oplus \mathfrak{u}=\{X \in \mathfrak{l}:$ ad $X(\mathfrak{g}) \subset \mathfrak{g}\}$ and then (1.7) follows from [2, Cor. 2.13]. For $X \in \mathfrak{g}_{1},(25)$ shows that $\nabla_{X}\left(\mathfrak{g}_{1}\right) \subset \mathfrak{g}_{1}$, $\nabla_{X}\left(\mathfrak{I}_{2}\right) \subset \mathfrak{I}_{2}$. Then, for $X, Y, Z \in \mathfrak{I}_{1}$,

$$
\begin{aligned}
& 2\left\langle\nabla_{X} Y, Z\right\rangle=\beta(P[X, Y], P Z)+\beta(P[Z, X], P Y)+\beta(P[Z, Y], P X) \\
&= \beta([P X, Q Y], P Z)+\beta([Q X, P Y], P Z)+\beta([P Z, Q X], P Y) \\
&+\beta([Q Z, P X], P Y)+\beta([P Z, Q Y], P X)+\beta([Q Z, P Y], P X) \\
&= \beta([P X, Q Y], P Z)+\beta([Q X, P Y], P Z)+\beta(P Z,[Q X, P Y]) \\
&-\beta(P X,[Q Z, P Y])-\beta(P Z,[P X, Q Y])+\beta([Q Z, P Y], P X) \\
&= 2\left\langle\left(P \mid \mathfrak{g}_{1}\right)^{-1}[Q X, P Y], Z\right\rangle
\end{aligned}
$$

SO

$$
\nabla_{X} Y=\left(P \mid \mathfrak{g}_{1}\right)^{-1}[Q X, P Y] \text { for } X, Y \in \mathfrak{g}_{1} .
$$

For $X \in \mathfrak{g}_{1}, Y, Z \in \mathfrak{s}_{2}$, one has

$$
2\left\langle\nabla_{X} Y, Z\right\rangle=\langle[X, Y], Z\rangle+\langle[Z, X], Y\rangle
$$


since $\langle[Z, Y], X\rangle=0$ by (1.6.1). Thus

$$
\nabla_{X} Y=\frac{1}{2}\left(\operatorname{ad}_{\mathfrak{s}_{2}} X-\left(\operatorname{ad}_{\mathfrak{s}_{2}} X\right)^{t}\right) Y \quad \text { for } X \in \mathfrak{s}_{1}, Y \in \mathfrak{s}_{2} .
$$

Let $\Sigma$ the projection of $g$ onto $\&$ with respect to the decomposition $\mathfrak{g}=\mathfrak{g} \oplus \mathfrak{f}$. As in [2, Prop. 2.9], one has

$$
\lambda(Z)=\Sigma \circ \operatorname{ad}_{3} Z \quad \text { for } Z \in \mathfrak{H} .
$$

In particular, for $X \in \mathfrak{g}_{1}$, we can apply (40) to $Z=Q X \in \mathfrak{u}_{1} \subset \mathfrak{f}$. For $Y \in \mathfrak{I}_{1}$, we have $[Q X, Y] \in \mathfrak{h}_{1}$ and $P\left\{[Q X, Y]-\left(P \mid \mathfrak{I}_{1}\right)^{-1} P[Q X, Y]\right\}=0$ so

$$
\Sigma([Q X, Y])=\left(P \mid \mathfrak{g}_{1}\right)^{-1} P[Q X, Y]=\left(P \mid \mathfrak{g}_{1}\right)^{-1}[Q X, P Y]=\nabla_{X} Y
$$

from (38). For $Y \in \mathfrak{s}_{2}$, we have $[Q X, Y] \in \mathfrak{g}_{2}$ so

$$
\begin{aligned}
\Sigma[Q X, Y] & =[Q X, Y]=\frac{1}{2}\left(\operatorname{ad}_{\mathfrak{\xi}_{2}} X+\operatorname{ad}_{\mathfrak{\xi}_{2}} \theta X\right) Y \\
& =\frac{1}{2}\left(\operatorname{ad}_{\mathfrak{\vartheta}_{2}} X-\left(\operatorname{ad}_{\mathfrak{s}_{2}} X\right)^{t}\right) Y=\nabla_{X} Y
\end{aligned}
$$

by (40). Thus

$$
\lambda(Q X)=\nabla_{X} \text { for } X \in \mathfrak{s}_{1} .
$$

Now let $a_{1}$ be the maximal abelian subspace of $p_{1}$ corresponding to the Iwasawa decomposition $\mathfrak{u}_{1} \oplus \mathfrak{g}_{1}$. Then $\operatorname{Ker}\left(Q \mid \mathfrak{g}_{1}\right)=a_{1}$ and $\operatorname{Im}\left(Q \mid \mathfrak{g}_{1}\right)$ $=l_{1}$, as is well known. With (41), this proves (1.8).

Next we consider $\mathfrak{x}:=\left\{X \in \mathfrak{g} ; \nabla_{X} \in \lambda(\mathfrak{f})\right\}$. From (1.8) we get $\mathfrak{n}_{1} \subset \mathfrak{x}$. Moreover, (41) shows $\nabla_{X}=0$ for $X \in \mathfrak{a}_{1}:=\mathfrak{g}_{1} \ominus \mathfrak{n}_{1}$. Hence $\mathfrak{g}_{1} \subset \mathfrak{x}$. Put $\mathfrak{x}_{2}:=\left\{X \in \mathfrak{g}_{2} ; \nabla_{X} \mathfrak{b}_{1}=0, \nabla_{X}\right.$ is a skew-symmetric derivation of $\left.\mathfrak{g}_{2}\right\}$. We claim

$$
\mathfrak{x}=\mathfrak{g}_{1}+\mathfrak{x}_{2} .
$$

Clearly $\mathfrak{x}_{2} \subset \mathfrak{x}$. Let now $X \in \mathfrak{g}_{2}, \nabla_{X} \in \lambda(\mathfrak{f})$. By (b) of the above remark we know $\nabla_{X}=\nabla_{n}+\operatorname{ad}(m+n)$ where $n \in \mathfrak{n}_{1}, m \in \mathfrak{m}_{1}, u \in \mathfrak{u}$. Hence $\nabla_{(X-n)}=\operatorname{ad}(m+u)$ is a skew-symmetric derivation of $\mathfrak{g}$ that annihilates $a_{1}$. From the definition of $\nabla_{Y}$ we derive that the $\mathfrak{g}_{1}$-component of $\nabla_{(X-n)} X_{1}, X_{1} \in \mathfrak{S}_{1}$, is $\nabla_{-n} X_{1}$. Hence $\nabla_{n}$ is a skew-symmetric derivation of $\mathfrak{g}_{1}$. Therefore, by (41), $Q n \in l_{1}$ and $n=0$ follows. So we have $\nabla_{X}=$ $\operatorname{ad}(m+u)$. From the definition of $\nabla_{X}$ we derive $\nabla_{X} \mathfrak{g}_{1} \subset \mathfrak{g}_{2}$ whence ad $m \mathfrak{g}_{1} \subset \mathfrak{g}_{2}$. This implies $\left[m, \mathfrak{g}_{1}\right]=0$ and $m=0$ follows. Consequently $\nabla_{X}=$ ad $u$. This proves $X \in \mathfrak{x}_{2}$.

From now on, we consider the case of a homogeneous Siegel domain as in $\S 1$. The following result is immediate.

TheOREM 2. Let $D$ be a homogeneous Siegel domain with Bergman metric $g$. In the notation of $\S 1$, the hypotheses of Theorem 1 are satisfied with $b=i e, \mathfrak{h}_{1}=\mathfrak{h}_{s s}, \mathfrak{u}_{1}=\mathfrak{l}_{s s}=\mathfrak{f} \cap \mathfrak{h}_{s s}, \mathfrak{g}_{1}=\mathfrak{g}_{s s}, \mathfrak{g}_{2}=\mathfrak{g}_{r}$, and $\mathfrak{u}=\mathfrak{t}_{a}$. 
For now, we reserve the notation $\mathfrak{g}, \nabla, \lambda$, etc. for the specific normal $j$-algebra and related objects constructed in $\S 1$. Suppose $\hat{S}$ is any simply-transitive split-solvable subgroup of $G$ with corresponding normal $j$-algebra $\hat{\mathfrak{s} \subset} \subset$ g. By [28, p. 46], we know $S$ and $\hat{S}$ are conjugate by an element of $G$. Since $G=K S$, we may assume $\hat{S}=k_{0} S k_{0}^{-1}$ for some $k_{0} \in K$. Let $\hat{\mathfrak{n}}=[\hat{\xi}, \hat{\mathfrak{g}}]$ with orthogonal complement $\hat{\mathfrak{a}}$, let $\hat{\nabla}$ be the covariant derivative operator induced on $\hat{\mathfrak{S}}$, let $\hat{\lambda}: \mathfrak{f} \rightarrow \mathfrak{S o}(\hat{\mathfrak{I}})$ be the induced isotropy representation, etc. One easily checks that

Ad $k_{0}: \mathfrak{g} \rightarrow \mathfrak{S}$ is a Lie algebra isomorphism preserving inner products and $j$ operators, hence also roots and root spaces

$$
\begin{gathered}
\hat{\lambda}\left(\operatorname{Ad} k_{0}(X)\right)=\operatorname{Ad} k_{0} \circ \lambda(X) \circ \operatorname{Ad}\left(k_{0}^{-1}\right) \mid \hat{\xi} \quad \text { for } X \in \mathfrak{H} \\
\text { Ad } k_{0} \circ \nabla_{X} \circ \operatorname{Ad~} k_{0}^{-1}=\hat{\nabla}_{\operatorname{Ad} k_{0}(X)} \text { for } X \in \mathfrak{g} .
\end{gathered}
$$

Let $\hat{\mathfrak{m}}=\{X \in \mathfrak{f}:$ ad $X(\hat{\mathfrak{S}}) \subset \mathfrak{s}\}$ and let $\hat{\mathfrak{D}}$ be the algebra of skew-symmetric derivations on $\hat{\mathfrak{y}}$. From [2, Cor. 2.13], we know $\hat{\lambda}$ maps $\hat{\mathfrak{m}}$ onto $\hat{\mathfrak{b}}$. It is also easy to see that $\operatorname{Ad} k_{0}$ maps $\mathfrak{m}$, resp. $\mathfrak{n}_{1}=\mathfrak{n}_{s s}$, resp. $\mathfrak{a}$, onto $\hat{\mathfrak{m}}$, resp. $\hat{\mathfrak{n}}_{1}$, resp. $\hat{a}$, and that

$$
\hat{\mathfrak{d}} \subset \mathfrak{g}_{0} .
$$

Since the action of $K$ on $D$ is by holomorphic maps, we also have

Each $T \in \hat{\mathfrak{b}}$ commutes with $j$.

For $T \in \hat{\mathfrak{b}}$, we get $T(\hat{\mathfrak{n}}) \subset \hat{\mathfrak{n}}$, hence $T(\hat{\mathrm{a}}) \subset \hat{\mathrm{a}}$. For any $\operatorname{root} \alpha, Y \in \hat{\mathfrak{n}}_{\alpha}$, and $H \in \hat{a}$, we get

$$
\begin{aligned}
\alpha(H) T Y_{\alpha} & =T\left[H, Y_{\alpha}\right]=\left[T H, Y_{\alpha}\right]+\left[H, T Y_{\alpha}\right] \\
& =\alpha(T H) Y_{\alpha}+\sum_{\beta} \beta(H)\left(T Y_{\alpha}\right)_{\mathfrak{n}_{\beta}},
\end{aligned}
$$

which implies

$$
T \mid \hat{a}=0 \quad \text { and } \quad T\left(\hat{\mathfrak{n}}_{\alpha}\right) \subset \hat{\mathfrak{n}}_{\alpha} \text { for } T \in \hat{d} .
$$

As in [3], one has

$$
\hat{\nabla}_{X} \equiv 0 \Leftrightarrow \hat{\nabla}_{X} \mid \hat{a} \equiv 0 \Leftrightarrow X \in \hat{a} .
$$

In particular, no nontrivial $\hat{\nabla}_{X}$ is in $\hat{\mathfrak{b}}$. Let $\hat{\mathfrak{n}}_{1}=\left\{X \in \hat{\mathfrak{n}}: \nabla_{X} \in \hat{\lambda}(\mathfrak{f})\right\}$.

TheOREM 3. Let $D$ be a homogeneous Siegel domain with Bergman metric and $\hat{\mathfrak{S}}$ any corresponding normal j-algebra with $\omega$ the form on $\hat{\mathfrak{S}}$ associated to the Bergman metric by $\omega[j X, Y]=\langle X, Y\rangle$. Then $\mathfrak{s}$ is the orthogonal direct sum of a subalgebra $\mathfrak{S}_{1}$ and an ideal $\hat{\mathfrak{S}}_{2}$ where $\hat{\mathfrak{S}}_{i}=\hat{\mathrm{a}}_{i} \oplus \hat{\mathrm{n}}_{i}$, $\hat{\mathrm{a}}_{i}=\hat{\mathrm{a}} \cap \hat{\mathfrak{S}}_{i}, \hat{\mathrm{n}}_{i}=\left[\hat{\mathfrak{S}}_{i}, \hat{\mathfrak{S}}_{i}\right]=\hat{\mathrm{n}} \cap \hat{\mathfrak{S}}_{i}\left(\hat{\mathrm{n}}_{1}\right.$ defined as above $)$ and

$$
\text { (3.1) } \hat{\lambda}(\mathfrak{f})=\hat{\mathfrak{D}} \oplus\left\{\hat{\nabla}_{X}: X \in \hat{\mathfrak{n}}_{1}\right\}
$$


(3.2) $\exp \hat{\xi}_{1} \cdot($ ie) is a totally geodesic Riemannian locally symmetric submanifold (so in particular, $X, Y \in \hat{\mathfrak{s}}_{1}$ implies $\hat{\nabla}_{X} Y \in \hat{\mathfrak{s}}_{1}$ )

(3.3) $\hat{\mathrm{n}}_{1}=\sum_{\alpha} \hat{\mathrm{n}}_{\alpha}$ where the sum is over the (nonzero) roots $\alpha$ such that $\alpha \mid \hat{\mathrm{a}}_{2}=0$ and $\hat{\mathrm{a}}_{2}$ is the maximal subspace of $\hat{\mathrm{a}}$ with this property

(3.4) For $k<l, 0 \neq \hat{n}_{\left(\varepsilon_{k}+\varepsilon_{l}\right) / 2} \subset \hat{n}_{1} \Rightarrow \hat{n}_{\left(\varepsilon_{k}-\varepsilon_{l}\right) / 2} \oplus \hat{n}_{\varepsilon_{k}} \oplus \hat{n}_{\varepsilon_{l}} \subset \hat{n}_{1}$

(3.5) $0 \neq \hat{\mathfrak{n}}_{\varepsilon_{k} / 2} \subset \hat{\mathrm{n}}_{1} \Rightarrow \hat{\mathrm{n}}_{\varepsilon_{k}} \subset \hat{\mathrm{n}}_{1}$

(3.6) $\hat{\mathrm{n}}_{\varepsilon_{k}} \subset \hat{\mathrm{n}}_{1} \Rightarrow \hat{\mathrm{n}}_{\varepsilon_{k} / 2} \oplus \sum_{l>k} \hat{\mathrm{n}}_{\left(\varepsilon_{k} \pm \varepsilon_{l}\right) / 2} \subset \hat{\mathrm{n}}_{1}$, although some of the spaces may be trivial

(3.7) For $k<l, 0 \neq \mathfrak{n}_{\left(\varepsilon_{k}-\varepsilon_{l}\right) / 2} \subset \hat{\mathfrak{n}}_{1} \Rightarrow \omega\left(X_{k}\right)=\omega\left(X_{l}\right)$.

REMARK. From (42)-(44), we may pull everything back to $\Xi$. It is only important to know the values of the form $\omega$ on the elements $X_{l} \in \mathfrak{n}_{\varepsilon_{l}}$ defined in (15); in fact $\omega$ must vanish on the orthogonal complement of $\mathfrak{a} \oplus j \mathfrak{a}$. The values of $\omega$ on $X_{l}$ may be determined from [3, Theorem 4]. With our present normalization, one has

$$
\omega\left(X_{l}\right)=\frac{1}{4} \boldsymbol{\sigma}\left(d_{l}, d_{l}\right)=\frac{1}{4} \boldsymbol{\sigma}\left(e, d_{l}\right) \text { and } \boldsymbol{\sigma}(e, e)=\operatorname{dim}_{\mathbf{R}} \mathscr{V}^{\mathbf{C}}+\operatorname{dim}_{\mathbf{C}} \mathscr{U} \text {. }
$$

The last equality was given incorrectly in $[11,12,13]$.

Proof. The first assertions and (3.1)-(3.3) are obvious from Theorem 2 and (42)-(48). The main problem with the rest is in comparing the indexing of the roots of $\mathfrak{g}$ with those of $\mathfrak{s}$ (although the roots themselves are related by $\left.\hat{\lambda}=\lambda \circ\left(\operatorname{Ad~} k_{0}\right)^{-1}\right)$. However, it is clear that roots of the form $\varepsilon_{m}$, resp. $\frac{1}{2} \varepsilon_{m}$, resp. $\frac{1}{2}\left(\varepsilon_{m}-\varepsilon_{n}\right)$, resp. $\frac{1}{2}\left(\varepsilon_{m}+\varepsilon_{n}\right)$ correspond. Thus if $0 \neq \hat{n}_{\left(\varepsilon_{k}+\varepsilon_{1}\right) / 2} \subset \hat{n}_{1}$, then the corresponding nonzero space $\mathfrak{n}_{\left(\varepsilon_{m}+\varepsilon_{n}\right) / 2}$ must be in $n_{1}=\mathfrak{n}_{s s}$, hence in $\mathfrak{g}_{-1}^{\prime \prime}$, which implies $d_{m}, d_{n} \in \mathscr{P}_{1}$. This gives $\mathfrak{n}_{\left(\varepsilon_{m}+\varepsilon_{n}\right) / 2} \oplus \mathfrak{n}_{\varepsilon_{m}} \oplus \mathfrak{n}_{\varepsilon_{n}} \subset \mathfrak{n}^{\prime \prime} \subset \mathfrak{n}_{s s}$. Transferring back to $\dot{\mathcal{S}}$ gives (3.4). Similar arguments give (3.5) and (3.6).

To prove (3.7), we again consider the corresponding nonzero space $\mathfrak{n}_{\left(\varepsilon_{m}-\varepsilon_{n}\right) / 2} \subset \mathfrak{n}_{1}=\mathfrak{n}_{s s}$, whose elements must be of the form $\left(T\left(b_{m n}\right), \hat{T}\left(b_{m n}\right)\right), m<n, b_{m n} \in \mathscr{X}$, by (21). From the definition of $\mathscr{B}_{i j}$, we know $d_{m}, d_{n} \in \mathscr{X}$ and $d_{m} X=\frac{1}{2} X=d_{n} X$ for $X \in \mathscr{B}_{m n}$. Moreover $X^{2}=\alpha d_{m}+\beta d_{n}, \alpha, \beta$ real, for such $X$. As $\mathscr{X}$ is a Jordan algebra, we know $X^{2} X^{2}=X\left(X X^{2}\right)$ and a simple computation now gives $\alpha=\beta$. Therefore, choosing $0 \neq X \in \mathscr{B}_{m n}$, we have

$$
\alpha \sigma\left(d_{n}, d_{n}\right)=\sigma\left(d_{n}, X^{2}\right)==\frac{1}{2} \sigma(X, X)>0
$$

and

$$
\alpha \sigma\left(d_{m}, d_{m}\right)=\sigma\left(d_{m}, X^{2}\right)=\frac{1}{2} \sigma(X, X)>0
$$

whence $\alpha>0$ and $\sigma\left(d_{m}, d_{m}\right)=\sigma\left(d_{n}, d_{n}\right)$. From the remark above, we get $\omega\left(X_{m}\right)=\omega\left(X_{n}\right)$. 
Remark. Properties (45)-(47) and Theorem 3 hold for any of the admissible Kähler metrics on $\hat{S}$ (i.e. left invariant metrics obtained from a form $\omega$ by $\langle X, Y\rangle=\omega[j X, Y])$, not just the Bergman metric. A direct proof can be given by computations within the normal $j$-algebra $\hat{\xi}$ using [2, Prop. 28]. For example (3.7) may be proved by computing $0=$ $\left\langle\left(\hat{\nabla}_{Z} R\right)(j X, j Z) j Z, Z\right\rangle$ where $Z \in \hat{\mathrm{n}}_{\left(\varepsilon_{k}-\varepsilon_{l}\right) / 2} \subset \hat{\mathrm{n}}_{1}$. Another proof can probably be obtained by generalizing the work of the second author.

Theorem 4. Let $D$ be a homogeneous Siegel domain with Bergman metric and suppose $\mathfrak{s}$ is an associated normal $j$-algebra satisfying

$$
\mathfrak{g}_{-1} \oplus \mathfrak{g}_{-1 / 2} \subset \mathfrak{g} \subset \mathfrak{g}_{-1} \oplus \mathfrak{g}_{-1 / 2} \oplus \mathfrak{g}_{0}=\underline{\operatorname{aff}}(D) .
$$

Then the isotropy representation $\hat{\lambda}: \mathfrak{f} \rightarrow \mathfrak{g} \mathfrak{D}(\mathfrak{g})$ is given on $\mathfrak{f}_{0}^{\perp}($ see $(7))$ by

$$
\hat{\lambda}\left(X_{-1}[a]+X_{1}[a]\right)^{+}=2 \hat{\nabla}_{X_{-1}[a]^{+}} \text {for } a \in \mathscr{P}_{1}
$$

$$
\hat{\lambda}\left(X_{-1 / 2}[d]+X_{1 / 2}[d]\right)^{+}=2 \hat{\nabla}_{X_{-1 / 2}[d]^{+}} \text {for } d \in \mathscr{P}_{1 / 2} .
$$

Further $\hat{\lambda}\left(k_{0}\right)=\hat{\mathfrak{D}} \oplus\left\{\hat{\nabla}_{X}: X \in \hat{\mathfrak{n}}_{1} \cap \mathrm{g}_{0}\right\}$.

Proof. Let $W=\left(X_{-1}[a]+X_{1}[a]\right)^{+}$. For $X_{-1}[c]^{+} \in g_{-1}$, (37) implies that

$$
\begin{aligned}
\hat{\lambda}(W)\left(X_{-1}[c]^{+}\right) & =-\left.\left[W^{*}, X_{-1}[c]\right]\right|_{i e}=\left.X_{0}\left(2 L_{a}(c), \phi(c) \phi(a)\right)\right|_{i e} \\
& =2 i L_{a}(c) e=2 i a c .
\end{aligned}
$$

Now by (25) $\hat{\nabla}_{X_{-1}[a]^{+}} X_{-1}[c]^{+}$is in $g_{0}$ so we may set $\hat{\nabla}_{X_{-1}[a]^{+}} X_{-1}[c]^{+}=$ $X_{0}(T, \hat{T})^{+}$. Then by $(25)$

$$
\begin{aligned}
\frac{1}{4} \sigma(T e, U e)=\left\langle X_{0}(T, \hat{T})^{+}, X_{0}(U, \hat{U})^{+}\right\rangle \\
\quad=\left\langle\hat{\nabla}_{X_{-1}[a]^{+}} X_{-1}[c]^{+}, X_{0}(U, U)^{+}\right\rangle \\
\quad=\frac{1}{2}\left\{\left\langle X_{-1}[U a]^{+}, X_{-1}[c]^{+}\right\rangle+\left\langle X_{-1}[a]^{+}, X_{-1}[U c]^{+}\right\rangle\right\} \\
\quad=\frac{1}{8}\{\sigma(U a, c)+\sigma(a, U c)\}=\frac{1}{4} \sigma(L(U e) a, c)=\frac{1}{4} \sigma(U e, a c),
\end{aligned}
$$

where at the end, we use the formula for $U+U^{t}$ given after (33). Thus $T e=a c$, hence $\left.X_{0}(T, \hat{T})\right|_{i e}=i a c$. This proves (49) on the subspace $g_{-1}$.

With the same $W$ but $X_{-1 / 2}[c]^{+} \in \mathfrak{g}_{-1 / 2}$, (37) now implies that

$$
\begin{aligned}
\hat{\lambda}(W)\left(X_{-1 / 2}[c]^{+}\right) & =-\left.\left[W^{*}, X_{-1 / 2}[c]\right]\right|_{i e}=\left.X_{1 / 2}[-i \phi(a) c]\right|_{i e} \\
& =i \phi(i e)(-i \phi(a) c)=i \phi(a) c .
\end{aligned}
$$


Now by $(25), \hat{\nabla}_{X_{-1}[a]^{+}} X_{-1 / 2}[c]^{+}$is in $g_{-1 / 2}$ so we may set $\hat{\nabla}_{X_{-1}[a]^{+}} X_{-1 / 2}[c]^{+}$ $=X_{-1 / 2}[d]^{+}$. Then by $(25)$

$$
\begin{aligned}
\operatorname{Re} \rho & (d, f)=\left\langle X_{-1 / 2}[d]^{+}, X_{-1 / 2}[f]^{+}\right\rangle \\
& =\left\langle\hat{\nabla}_{X_{-1}[a]^{+}} X_{-1 / 2}[c]^{+}, X_{-1 / 2}[f]^{+}\right\rangle \\
& =\frac{1}{2}\left\langle\left[X_{-1 / 2}[f]^{+}, X_{-1 / 2}[c]^{+}\right], X_{-1}[a]^{+}\right\rangle \\
& =\frac{1}{2}\left\langle X_{-1}[4 \operatorname{Im} F(f, c)]^{+}, X_{-1}[a]^{+}\right\rangle \\
& =\frac{1}{2} \sigma(\operatorname{Im} F(f, c), a)=\frac{1}{2} \operatorname{Re} \rho(i \phi(a) c, f) .
\end{aligned}
$$

Thus $d=\frac{1}{2} i \phi(a) c$, hence $\left.X_{-1 / 2}[d]\right|_{l e}=\frac{1}{2} i \phi(a) c$. This proves (49) on the subspace $g_{-1 / 2}$.

Since both sides of (49) commute with $j$ and $j\left(\mathfrak{g}_{-1}\right)=\hat{s} \cap \mathfrak{g}_{0}$, this proves (49) on $\hat{s}$.

The proof of (50) is similar

As $\hat{\mathfrak{D}} \subset \mathfrak{f}_{0}$, whence $\hat{\lambda}(\hat{\mathfrak{D}})=\hat{\mathfrak{b}}$, the last statement is clear.

REMARK. If we consider a vector field $X$ on $D$ as a function from $D$ to $\mathscr{V}^{\mathrm{C}} \oplus \mathscr{U}$, then we may take its differential $d_{i e} X$ at the point $i e$, which will be a linear transformation on $T_{i e} D=T_{i e}\left(\mathscr{V}^{\mathbf{C}} \oplus \mathscr{U}\right)$ for $X \in \mathfrak{H}^{*}$. With this interpretation, $\hat{\lambda}\left(X^{+}\right)=d_{i e} X$. This gives an alternate way of doing some of the calculations of the proof above.

In the case where $\mathfrak{g}$ is a normal $j$-algebra of a homogeneous Siegel domain we can give a more precise description of

$$
\mathfrak{x}=\left\{X \in \hat{\xi} ; \nabla_{X} \in \lambda(\mathfrak{t})\right\} .
$$

As a consequence of Theorem 1 we have shown $\mathfrak{x}=\mathfrak{g}_{1}+\mathfrak{x}_{2}$ where $\mathfrak{x}_{2} \subset \hat{s}_{2}$ consists of $X \in \hat{s}_{2}$ so that $\nabla_{X}$ is a skew-symmetric derivation of $\hat{\mathfrak{s}}$. From (48) we get $\mathfrak{x}_{2}=\mathfrak{a}_{2}=\mathfrak{g}_{2} \ominus\left[\hat{\mathfrak{y}}_{2}, \hat{\mathfrak{s}}_{2}\right]$ whence $\mathfrak{x}=\hat{\mathfrak{y}}_{1}+\mathfrak{a}_{2}$.

\section{Applications of the isotropy representation.}

Theorem 5. Let $D$ be a homogeneous Siegel domain with Bergman metric. Then the almost complex structure map is in the image of the infinitesimal isotropy representation if and only if the domain is symmetric.

Proof. Let $b$ be a point of $D$ and $\lambda: \mathfrak{f} \rightarrow \mathfrak{b} \mathfrak{0}\left(T_{b} D, g_{b}\right)$ the infinitesimal isotropy representation and let $j: T_{b} D \rightarrow T_{b} D$ be the almost complex structure map at $b$. Let $S$ be any simply-transitive Lie subgroup of $G$ with corresponding subalgebra $\mathfrak{I}$ (not necessarily the $S, \mathfrak{g}$ of $\S 1$ ). Identify $\mathfrak{g}$ with $T_{b} D$ as usual. For any skew-symmetric operator $L: \mathfrak{g} \rightarrow \mathfrak{g}$ and any $X \in \mathfrak{g}$, let

$$
C_{X}(L)=\left[L, \nabla_{X}\right]-\nabla_{L X}
$$


where the operator $\nabla_{X}$ on $\mathfrak{g}$ is defined by $(25)$. By $[2 ; 2.8], \lambda(\mathfrak{f})$ is the largest subspace of skew-symmetric operators of $\xi$ whose elements annihilate the curvature tensor and which is invariant under all $C_{X}$. Clearly $j$ is a skew-symmetric operator which annihiliates the curvature tensor but (using the Kähler property) $j \in \lambda(\mathfrak{f})$ implies $-\nabla_{j X}=\left[j, \nabla_{X}\right]-\nabla_{j X}=$ $C_{X}(j) \in \lambda(\mathfrak{f})$, for all $X \in \mathfrak{S}$. Therefore $\nabla_{Y} \in \lambda(\mathfrak{f})$ for all $Y \in \mathfrak{S}$ whence $\left(\nabla_{Y} R\right)=0$ for all $Y \in \mathfrak{S}$ and $D$ is symmetric.

Note that the proof only uses that $g$ is an $S$-invariant Kähler metric.

The converse is well known.

REMARK. Let $\mathfrak{I}_{-1 / 2}=\oplus \mathfrak{n}_{\varepsilon_{k} / 2}$. Let $T: \mathfrak{g} \rightarrow \mathfrak{g}$ be defined by $T \mathfrak{I}_{-1 / 2}=$ $j \mid \mathfrak{S}_{-1 / 2}$ and $T \mid\left(\mathfrak{S}_{-1 / 2}\right)^{\perp}=0$. Then an application of the Kähler condition (see [3, p. 64]) shows that $T$ is always a skew-symmetric derivation of $\mathscr{g}$. In fact, $T$ is the skew-symmetric derivation induced from $(0, i$ Id $) \in \mathfrak{g}_{0}$ which is obviously in $\mathfrak{f}$ (and even in $\mathfrak{m}$ ). We shall denote this derivation by $\left.j\right|_{-1 / 2}$ or similar notation.

TheOREM 6. Let $D$ be a homogeneous Siegel domain. Then the dimension of the orbit of the origin 0 under $K$ equals the dimension of the Silov boundary $B$ if and only if $D$ is symmetric.

Proof. Using the notations and constructions of $\S 1$, an element $Y^{*}$ of $\mathfrak{F}^{*}$ is of the form $\left(X_{-1}[a]+X_{1}[a]\right)+\left(X_{1 / 2}[d]+X_{-1 / 2}[d]\right)+X_{0}(T, \hat{T})$ where $a \in \mathscr{P}_{1}, d \in \mathscr{P}_{1 / 2},(T, \hat{T}) \in \mathrm{g}_{0}$ with $T e=0$ (see (7)). From (2)-(6), one has $\left.Y^{*}\right|_{0}=a+d$ so

$$
\left\{\left.Y^{*}\right|_{0}: Y \in \mathfrak{F}\right\}=\mathscr{P}_{1}+\mathscr{P}_{1 / 2}
$$

and $\operatorname{dim} K \cdot 0=\operatorname{dim} \mathscr{P}_{1}+\operatorname{dim} \mathscr{P}_{1 / 2}$ while $\operatorname{dim} B=\operatorname{dim} \mathscr{V}+\operatorname{dim} \mathscr{U}$. Thus $\operatorname{dim} B=\operatorname{dim} K \cdot 0$ implies $\operatorname{dim} \mathscr{P}_{1}=\operatorname{dim} \mathscr{V}$ and $\operatorname{dim} \mathscr{P}_{1 / 2}=\operatorname{dim} \mathscr{U}$. By [21, Th. 4, p. 484], this implies $\mathfrak{r}_{-1}=\mathfrak{r}_{-1 / 2}=0$ which in turn $\mathfrak{r}_{0}=0$ since $\left[\mathfrak{r}_{0}, \mathfrak{g}_{-1}\right] \subset \mathfrak{r}_{-1}$. But radical $r=0$ implies $D$ is symmetric. The converse is well known.

REMARK. We consider one reason to study $K \cdot 0$. Let $S(\eta, \xi)$ be the Szegö kernel of $D$ and let $P_{\eta}(\xi)=P(\eta, \xi)=|S(\eta, \xi)|^{2} / S(\xi, \xi), \eta \in B$, $\xi \in D$ be the Poisson kernel (see Gindikin [16], Koranyi-Stein [24], and Koranyi [23]). Let $\Delta$ be the Laplacian of the Bergman metric. Koranyi [23] has shown that $\Delta P_{\eta} \equiv 0$ for all $\eta \in B$ if $D$ is symmetric, Xu Yichao [33] has shown the converse. Our interest is in the set $\{(\eta, \xi) \in B \times D$ : $\left.\left(\Delta P_{\eta}\right)(\xi)=0\right\}$ or, less ambitiously, the set

$$
B_{p}=\left\{\eta \in B:\left(\Delta P_{\eta}\right)(b)=0\right\}
$$


where $b=i e$ is our base point. Suppose $D$ is a tube domain. Then we have checked that $\left(\Delta P_{0}\right)(b)=0$ (even this can fail for a non symmetric, non tube domain). Further, it is easy to see that the square of the Szegö kernel is a constant multiple of the Bergman kernel in this case. Using known invariant properties of the Bergman kernel, one has $P_{g \eta}(g \xi)=$ $\mu(g, \eta) P_{\eta}(\xi)$ for $\eta \in B, \xi \in D, g \in G$ (when $g \cdot \eta$ is defined); the point is that $\mu(g, \eta)$ does not depend on $\xi$. For $k \in K$, since $\Delta$ is preserved by the isometry $k$, one gets

$$
\begin{aligned}
\left(\Delta P_{k \cdot 0}\right)(b) & =\left(\left(\Delta P_{k \cdot 0}\right) \circ k\right)(b)=\left(\Delta\left(P_{k \cdot 0} \circ k\right)\right)(b) \\
& =\mu(k, 0)\left(\Delta P_{0}\right)(b)=0 .
\end{aligned}
$$

Thus $B_{p} \supset K \cdot 0$. We have computed examples to show that $B_{p}$ can be significantly larger than $K \cdot 0$.

REMARK. Even in the symmetric case, the action of $K$ on $B$ is not globally defined if one uses the unbounded (Siegel) domain realization rather than the bounded domain realization. However

$$
\mathfrak{f}^{*}=\left(\mathfrak{f}^{*} \cap\left(\mathfrak{g}^{\prime \prime}\right)^{*}\right) \oplus\left(\operatorname{Der}\left(\mathscr{X} \ominus \mathscr{P}_{1}\right)\right) \oplus \mathfrak{t}_{a}^{*}
$$

is a direct sum of Lie algebras so the isotropy group $K$ at $i e$ is a product of three commuting groups. It is known (e.g. [21, Th. 10]) that each biholomorphic map of $D$ is birational. Then an element $g \in K$ can be written as a product $g=g_{1} g_{2} g_{3}$ where each $g_{i}$ is birational and $g_{2} g_{3}$ is in fact linear. Here $g_{1} \in \exp \left(\mathfrak{f} \cap \mathfrak{g}^{\prime \prime}\right), g_{2} \in \exp \left(\operatorname{Der}\left(\mathscr{X} \ominus \mathscr{P}_{1}\right)\right), g_{3} \in \exp t_{a}$. One defines

$K \cdot 0=\{g(0): g \in K$ and the denominator of $g$ does not vanish at 0$\}$ where clearly the denominator of $g$ does not vanish at 0 if and only if the same is true for $g_{1}$ and, in that case, $g(0)=g_{1}(0)$. We claim that the denominator of $g_{1}$ vanishes at 0 if and only if the denominator of $g_{1} \mid \mathscr{P}_{1}^{\mathbf{C}} \times \mathscr{P}_{1 / 2}$ vanishes at 0 . Now a theorem of Kaup (cf. [20, Th. 6.1]) says Aut $D=\mathscr{T} \cdot \mathscr{N} \cdot \mathrm{GL}(D) \cdot \mathscr{T}$ where $\mathscr{T}=\exp \left(\mathfrak{g}_{-1} \oplus \mathfrak{g}_{-1 / 2}\right), \quad \mathscr{N}=$ $\exp \left(\mathfrak{g}_{-1 / 2} \oplus \mathfrak{g}_{1}\right)$. It is clear we need only consider denominators for elements of $\mathscr{N}$. Using the notation and results of $\left[11\right.$, p. 557, $\left.\left(6.8^{\prime \prime}\right)\right]$, and the relation $\exp D_{w} m=m+\left(0, i \phi\left(m_{V}\right) w\right)$, we see that

$$
\begin{aligned}
g(m) & :=\exp X_{1 / 2}[w] \exp X_{1}[x](m) \\
& =\exp X_{1 / 2}[w]\left(m^{x}\right)=\exp D_{w}\left[\left(m^{x}\right)^{s}\right],
\end{aligned}
$$

where $s=w-i F(w, w)$. From a result of Loos [26, 8.16], we know that the denominator of a "quasi-inverse" $a^{b}$ is $N(a, b)$ as defined in $[26,16.9]$. Thus the denominator of $g(m)$ above is

$$
N\left(m^{x}, w-i F(w, w)\right)=N(m, x+w-i F(w, w)) N(m, x)^{-1}
$$


(cf. [26, 16.11]). Finally applying [26, 16.13] and [11, Th. 5.6], we see that the denominator of $g(m)$ just depends on the orthgonal projection of $m$ on $\mathscr{P}_{1}^{\mathbf{C}} \oplus \mathscr{P}_{1 / 2}$. Thus an element in $\mathscr{N}$ has the same denominator as its restriction to $\mathscr{P}_{1}^{\mathbf{C}} \oplus \mathscr{P}_{1 / 2}$. This proves our claim.

Combining the above claim with the action of $\exp \left(\mathfrak{f} \cap \mathfrak{g}^{\prime \prime}\right)$, we see that $K \cdot 0=K_{1} \cdot 0$, where $K_{1}$ is the isotropy group of the symmetric "subdomain" $D^{\prime}$ at the point $i e^{\prime}$, and $K_{1} \cdot 0$ is in turn precisely what is familiar from the symmetric case, namely, the Šilov boundary of $D^{\prime}$ which is just $\mathscr{P}_{1} \oplus \mathscr{P}_{1 / 2}$. Thus we have the following refinement of Theorem 6 ,

$$
K \cdot 0=\mathscr{P}_{1} \oplus \mathscr{P}_{1 / 2}=\text { Šilov boundary of } D^{\prime} \text {. }
$$

Now we consider the algebra of $G$ invariant differential operators (see [18, Ch. 10]). The problem of finding $G$ invariant first order operators obviously reduces to that of finding $G$ invariant first order operators without constant term, i.e. $G$ invariant vector fields. Let $S$ be any simply-transitive subgroup of $G$. The isometry $S \tilde{S} D$ implies that each left invariant vector field $Y$ on $S$ determines an $S$ invariant vector field, also denoted $Y$, on $D$ by

$$
\left.Y\right|_{s \cdot b}=\left.\frac{d}{d t}\right|_{t=0}(s \cdot \exp t Y \cdot b), \quad s \in S, Y \in \mathfrak{S}
$$

where $b$ is our chosen base point. Since $G=S K=K S$, to find $G$ invariant vector fields, we need only find those $Y \in \mathfrak{g}$ which are also $K$ invariant. Suppose $Y \in \mathfrak{g}$ and $\Lambda(k) Y_{b}=Y_{b}$ for all $k \in K$. For any point $\xi \in D$, we have $s \in S$ so that $s \cdot b=\xi$ and for any $k \in K$, we have $k_{1} \in K, s_{1} \in S$ with $k s=s_{1} k_{1}$ (and hence $\left.k \cdot \xi=s_{1} \cdot b\right)$. Thus

$$
k_{*}\left(Y_{\xi}\right)=k_{*}\left(Y_{s \cdot b}\right)=k_{*} s_{*}\left(Y_{b}\right)=\left(s_{1}\right)_{*}\left(k_{1}\right)_{*}\left(Y_{b}\right)=Y_{s_{1}(b)}=Y_{k \cdot \xi}
$$

so $Y$ is $K$ invariant. Finally, we note $K$ is connected. Treating the isotropy representations as represented on $\mathfrak{B}$, we get

$$
\begin{aligned}
\mathfrak{b} & =\{Y \in \mathfrak{g}: \lambda(\mathfrak{f}) Y=0\}=\{Y \in \mathfrak{g}: \Lambda(K) Y=Y\} \\
& =\text { set of } G \text { invariant vector fields on } D .
\end{aligned}
$$

REMARK. It is clear that $\mathfrak{b}$ is actually a $j$-invariant Lie subalgebra which, by (51) is closed under $(X, Y) \rightarrow \nabla_{X} Y$.

THEOREM 7. Let $D$ be a homogeneous Siegel domain with Bergman metric. Then there exist nontrivial $G$ invariant vector fields if and only if $D$ is not symmetric.

Proof. We use the notation and construction of Theorem 3 without the over symbol ^. If $\mathfrak{a}_{2}=0$, then (3.3) implies $\mathfrak{n}=\mathfrak{n}_{1}$, and (48) and [ 2 , 
Prop. 2.8] imply $\nabla R=0$. Hence if $D$ is nonsymmetric, we have $0 \neq$ $H \in \mathfrak{a}_{2}$ and for any skew-symmetric derivation $T$ of $\mathfrak{g}, T(H)=0$ by (46). On the other hand, if $\mathfrak{n}_{\alpha}$ is a root space in $\mathfrak{n}_{1}$ and $Y \in \mathfrak{n}_{\alpha}$, then (47) and (30) show that $\nabla_{Y} H=\nabla_{H} Y+[Y, H]=-\alpha(H) Y=0$. Thus $\lambda(\mathfrak{f}) \cdot H=0$ by Theorem 3 . By (55), $H$ is a $G$ invariant vector field on $D$.

The converse is well known since the algebra of $G$ invariant operators is generated by operators of even order in the symmetric case.

Now suppose a vector field $Y$ on $D$, considered as a first order differential operator, commutes with the Laplacian. Then the local oneparameter groups generating $Y$ leave the Laplacian invariant, hence must be isometries. Thus $Y$ is a Killing vector field.

LeMma. No nonzero vector field $Y \in \mathfrak{g}$ is a Killing vector field.

Proof. We will actually prove this for any normal $j$-algebra $\mathfrak{g}$ associated to $D$ and any admissible metric on $\mathfrak{g}$. If $Y \in \mathfrak{g}$ is Killing, then for all $V, W \in \mathfrak{g}$, one has $\left\langle V, \nabla_{W} Y\right\rangle=-\left\langle W, \nabla_{V} Y\right\rangle$ which implies

$$
\left\langle\nabla_{W} V, Y\right\rangle=-\left\langle\nabla_{V} W, Y\right\rangle, \quad V, W \in \mathfrak{s} .
$$

As in [3], let $X_{l}$ be the unique vector in $n_{\varepsilon_{l}}$ so that $\varepsilon_{l}\left(j X_{m}\right)=\delta_{l m}$. Choosing $Y=W=X_{l}$ and noting $\nabla_{X_{l}} X_{l}=j X_{l},(56)$ yields $0=\left\langle j X_{l}, Y\right\rangle$, so $Y \in \mathfrak{n}$. For any root $\alpha$, choosing $V=H_{\alpha}$ (the vector in a dual to $\alpha$ with respect to $\langle\rangle$,$) and W \in \mathfrak{n}_{\alpha},(56)$ yields $0=\left\langle\nabla_{W} H_{\alpha}, Y\right\rangle-\alpha\left(H_{\alpha}\right)\langle W, Y\rangle$ so $\langle W, Y\rangle=0$. Hence, $Y=0$.

TheOReM 8. Let D be a homogeneous Siegel domain. Then the algebra of $G$ invariant differntial operators is commutative if and only if $D$ is symmetric.

Proof. If $D$ is nonsymmetric, Theorem 7 implies there is a nontrivial $G$ invariant vector field $Y$. If $Y$ commutes with the Laplacian, it is Killing, which contradicts the Lemma. The converse is well known.

3.1. The set $\mathfrak{b}$ of $G$ invariant vector fields. In this subsection, we study the set $\mathfrak{b}$ defined in (55). We identify $\mathfrak{g}$ with $\mathscr{V}^{\mathbf{C}} \oplus \mathscr{U}$ (as vector spaces) so that $b \in \mathscr{V}$ corresponds to $\left(X_{-1}[b]\right)^{+} \in \mathfrak{g}_{-1}$ and $d \in \mathscr{U}$ corresponds to $\left(X_{-1 / 2}[d]\right)^{+} \in \mathfrak{g}_{-1 / 2}$. From the remark after Theorem 4 , we get $\mathfrak{b}=$ $\left\{z \in \mathscr{V}^{\mathbf{C}} \oplus \mathscr{U}: d_{i e} X(Z)=0\right.$ for all $\left.X \in \mathfrak{H}\right\}$. In particular, $z \in \mathfrak{b}$ implies $(T, \hat{T})(z)=0$ for all $(T, \hat{T}) \in \mathfrak{f}_{0}$. But $(0, i$ Id $) \in \mathfrak{f}_{0}$, whence $z \in \mathfrak{b}$

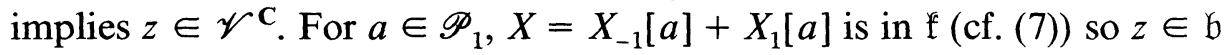
implies

$$
0=d_{i e} X(z)=\left(2 L_{a}(i e), \phi(i e) \phi(a)\right) z=2 L_{a}(i e) z=2 i a z
$$


Choosing $a=e^{\prime}$ gives $z \in \mathscr{L}_{0}\left(e^{\prime}\right)=\left\{w \in \mathscr{L}^{\mathbf{C}}: e^{\prime} w=0\right\}$. An easy computation now shows that

$$
\mathfrak{b}=\left\{z \in \mathscr{L}_{0}\left(e^{\prime}\right):(T, \hat{T}) z=0 \text { for all }(T, \hat{T}) \in \operatorname{Der}\left(\mathscr{X} \ominus \mathscr{P}_{1}\right) \oplus \mathrm{t}_{a}\right\} .
$$

We write $\mathscr{X} \ominus \mathscr{P}_{1}=\oplus_{k=1}^{g-1} \mathscr{C}_{k k}$ where the $\mathscr{C}_{k k}$ are the simple summands of $\mathscr{X} \ominus \mathscr{P}_{1}$. We put $c_{g}:=e^{\prime}, c_{k}:=$ unit of $\mathscr{C}_{k k}$ and consider the Peirce decomposition of $\mathscr{L}^{\mathbf{C}}$ relative to $c_{1}, \ldots, c_{g}, \mathscr{L}^{\mathbf{c}}=\oplus_{1 \leq i \leq j \leq g} \mathscr{C}_{i j}^{\mathbf{C}}$. For $z \in \mathfrak{b}$, the results above imply $z=\sum_{1 \leq i \leq j \leq g-1} z_{i j}$ where $z_{i j} \in \mathscr{C}_{i j}^{\mathbf{C}}$. Moreover $z_{k k}$ is annihilated by all derivations of $\mathscr{C}_{k k}$. But up to scalar multiple, the only vector with this property for all derivations of a simple formally real Jordan algebra is its identity. Thus $z_{k k} \in \mathbf{C} c_{k}$. It is easy to see that $\mathbf{C} c_{k}$ is actually contained in $\mathfrak{b}$. Thus

$$
\mathfrak{b}=\bigoplus_{k=1}^{g-1} \mathbf{C} c_{k} \oplus\left(\bigoplus_{1 \leq i \leq j \leq g-1}\left(g \cap \mathscr{C}_{i j}^{\mathbf{C}}\right)\right) .
$$

It is known ([7, Lemma 3.1] and [9, Th. 8.6]) that the map

$$
\mathscr{C}_{k k} \ni z \rightarrow 2 L(z) \mid \mathscr{C}_{k r} \in \operatorname{Sym}\left(\mathscr{C}_{k r},\langle,\rangle\right), \quad k \neq r,
$$

is a homomorphism of Jordan algebras. Also, Der $\mathscr{C}_{k k}$ is spanned by the commutators of left multiplications. The corresponding Lie algebra of skew-adjoint endomorphisms, extended $\mathbf{C}$ linearly to $\mathscr{C}_{k r}^{\mathbf{C}}$ will be denoted $\mathscr{D}_{k r}$.

LEMMA. If rank $\mathscr{C}_{k k} \geq 2$, then the only vector in $\mathscr{C}_{k r}^{\mathrm{C}}$ annihilated by $\mathscr{D}_{k r}$ is the zero vector.

Proof. We split $\mathscr{C}_{k r}^{\mathrm{C}}$ into irreducible subspaces relative to the given representation of the Jordan algebra. The space of vectors annihiliated by $\mathscr{D}_{k r}$ splits accordingly. Thus, we may assume $\mathscr{C}_{k r}^{\mathbf{C}}$ is irreducible. If $\mathscr{C}_{k k}=$ $\operatorname{Sym}(n, \mathbf{F})$, for $\mathbf{F}=\mathbf{R}, \mathbf{C}$, or $\mathbf{H}$ (Quaternions), then $\mathscr{C}_{k r}=\mathbf{F}^{n}$ and $L(z) u=$ $z u$. The commutators of the $L(z)$ contain at least all skew-symmetric real amtrices. These simultaneously annihilate no common vector if $n \geq 2$. The case $\mathscr{C}_{k k}=\operatorname{Sym}(3$, Cayley) does not occur so it remains to consider the case $\mathscr{C}_{k k}=\left[\mathscr{C}_{k k}, \mu, c_{k}\right]$. In this case, the representation $z \rightarrow \psi(z)=$ $L(z) \mid \mathscr{C}_{k r}$ satisfies $\psi(z)^{2}=\sigma(z, z)$ Id on $\left\{z \in \mathscr{C}_{k k}: \sigma\left(z, c_{k}\right)=0\right\}$. Thus $\psi(y) \psi(z)+\psi(z) \psi(y)=2$ Id if $\sigma(y, z)=0$. In particular $[\psi(y), \psi(z)]$ $=2 \psi(y) \psi(z)$ and this is invertible. So in this case, $\mathscr{D}_{k r}$ also annihilates no nonzero vector.

As an easy consequence of this Lemma, we get

$$
0 \neq \mathfrak{b} \cap \mathscr{C}_{k r}^{\mathbf{C}} \quad(k \neq r) \Rightarrow \mathscr{C}_{k k} \simeq \mathscr{C}_{r r} \simeq \mathbf{R} .
$$

Further, we note that $e-e^{\prime}$ and $T\left(e-e^{\prime}\right)$ are in $\mathfrak{b}$ and $\mathfrak{b}$ is a subalgebra of $\mathscr{L}^{\mathrm{C}}$. 
It is clear from the above that it is of great importance ot determine $\mathfrak{b}$ in case $\mathscr{X} \cong \mathbf{R} \oplus \mathbf{R}$. This corresponds - essentially - to all homogeneous Siegel domains over a circular cone. the second author hopes to pursue this topic in a forthcoming publication.

3.2. The center of $\mathfrak{f}$. In this subsection we collect some results on the structures of the center of $\mathfrak{f}$ which will lead to another characterization of the symmtric case. Actually we do our computations in the isomorphic algebra $\mathfrak{f}^{*}$ of vector fields on $D$ and use the splitting of (7) with the notation

$$
\begin{aligned}
\mathfrak{f}_{1}^{*} & =\left\{X_{-1}[b]+X_{1}[b]: b \in \mathscr{P}_{1}\right\} \\
\mathfrak{f}_{1 / 2}^{*} & =\left\{X_{-1 / 2}[d]+X_{1 / 2}[d]: d \in \mathscr{P}_{1 / 2}\right\} .
\end{aligned}
$$

We fix $Z \in z\left(\mathfrak{f}^{*}\right)=$ center( $\left.\mathfrak{f}^{*}\right)$ with $Z=Z_{1}+Z_{1 / 2}+Z_{0}, \quad Z_{1}=$ $X_{-1}[a]+X_{1}[a], \quad Z_{1 / 2}=X_{-1 / 2}[d]+X_{1 / 2}[d]$, and $Z_{0}=X_{0}(T, \hat{T})$ for some $a \in \mathscr{P}_{1}, d \in \mathscr{P}_{1 / 2}$, and $(T, \hat{T}) \in \mathrm{g}_{0}$ with $T e=0$.

Now $X_{0}(0, i \mathrm{Id})$ is always in $\mathfrak{f}_{0}^{*}$ (provided $\mathfrak{g}_{-1 / 2} \neq 0$ ) and we have (see $[12, \S 6]) 0=\left[Z, X_{0}(0, i\right.$ Id $\left.)\right]=\left[Z_{1 / 2}, X_{0}(0, i\right.$ Id $\left.)\right]=X_{-1 / 2}[i d]+X_{1 / 2}[i d]$. This implies $d=0$ and hence $Z_{1 / 2}=0$.

Now pick $Y=X_{-1}[b]+X_{1}[b] \in \mathfrak{H}_{1}^{*}$. Taking components of $0=$ $[Z, Y]$ with respect to the splitting (7) and again using the explicit bracket product formulas of $[12, \S 6]$, we get

$$
\begin{aligned}
0=\left[Z_{1}, Y\right] & =X_{0}\left(2 L_{b}(a), \phi(a) \phi(b)\right)-X_{0}\left(2 L_{a}(b), \phi(b) \phi(a)\right) \\
& =X_{0}(4[L(a), L(b)],[\phi(a), \phi(b)]) \\
0 & =\left[Z_{0}, Y\right]=-\left(X_{-1}[T b]+X_{1}[T b]\right) .
\end{aligned}
$$

Then (60) implies that $0=[L(a), L(b)]=[\phi(a), \phi(b)]$ for all $b \in \mathscr{P}_{1}$. In particular, standard facts of Jordan theory imply $a$ is in the center of $\mathscr{P}_{1}$. Further, (61) for all $Y$ is equivalent to $T \mid \mathscr{P}_{1}=0$.

For an arbitrary element $X_{0}=X_{0}(R, \hat{R}) \in \mathfrak{E}_{0}^{*}$, the condition $0=$ $\left[Z, X_{0}\right]$ implies $0=\left[Z_{1}, X_{0}\right]$ and $0=\left[Z_{0}, X_{0}\right]$, which in turn gives

$$
\begin{aligned}
& 0=R a \\
& 0=[T, R] .
\end{aligned}
$$

Condition (62) is automatically satisfied whenever $a$ is in the center of $\mathscr{P}_{1}$ and (63) is equivalent to the condition that $Z_{0}$ is in center $\left.\left(\mathfrak{f}_{0}^{*}\right)=z_{(\mathfrak{f}}^{*}\right)$.

Finally, for an arbitrary element $W=X_{-1 / 2}[w]+X_{1 / 2}[w] \in \mathfrak{f}_{1 / 2}^{*}$, we get

$$
0=[Z, W]=X_{-1 / 2}[i \phi(a) w-\hat{T} w]+X_{1 / 2}[i \phi(a) w-\hat{T} w]
$$


which is equivalent to

$$
\hat{T} w=i \phi(a) .
$$

Combining these results we have

$$
\begin{aligned}
& z\left(k^{*}\right)=\left\{X_{-1}[a]+X_{1}[a]+X_{0}(T, \hat{T}): a \in \operatorname{center}\left(\mathscr{P}_{1}\right),\right. \\
&\left.X_{0}(T, \hat{T}) \in{ }_{z}\left(k_{0}^{*}\right), T\left|\mathscr{P}_{1}=0, \hat{T}\right| \mathscr{P}_{1 / 2}=i \phi(a)\right\} .
\end{aligned}
$$

By (20) we know

$$
X_{0}(T, \hat{T})=X_{0}\left(T^{\prime \prime}, \hat{T}^{\prime \prime}\right)+\sum X_{0}\left(T_{j}, \hat{T}_{j}\right)+X_{0}\left(T_{t}, \hat{T}_{t}\right)
$$

where the summands are in the obvious subspaces of $z_{(}\left(\mathfrak{f}_{0}^{*}\right)$. From our conditions we obviously get $T^{\prime \prime} \mid \mathscr{P}_{1}=0$ and $\hat{T}^{\prime \prime} \mid \mathscr{P}_{1 / 2}=i \phi(a)$. Hence

$$
\begin{aligned}
& \text { center }\left(\mathfrak{f}^{*}\right)=\text { center } \mathrm{t}_{a}^{*} \oplus \sum \text { center } \mathfrak{f}_{0 j}^{*} \\
& \oplus\left\{X_{-1}[a]+X_{1}[a]+X_{0}(T, \hat{T}): a \in \text { center } \mathscr{P}_{1},\right. \\
& \left.X_{0}(T, \hat{T}) \in \text { center } \mathfrak{g}_{0}^{\prime \prime *}, T|\mathscr{X}=0, \hat{T}| \mathscr{P}_{1 / 2}=i \phi(a)\right\} \text {. }
\end{aligned}
$$

REMARK (a) It is clear that $X_{0}(T, \hat{T})$ in the last summand is uniquely determined. However, a more precise description requires a considerable effort that seems not to be appropriate for the purposes of this paper. rank 2 .

(b) center $\mathfrak{f}_{0 j}$ is nontrivial only if $\mathscr{L}_{j j}$ contains special simple ideals of

(c) It is clear that (66) splits canonically into the irreducible summands of a given homogeneous Siegel domain.

We note that to each a $\in \operatorname{center}\left(\mathscr{P}_{1}\right)$ there exists $X_{0}(T, \hat{T}) \in \mathrm{g}_{0}^{*}$ so that $X_{-1}[a]+X_{1}[a]+X_{0}(T, \hat{T}) \in \operatorname{center}\left(\mathfrak{f}^{*}\right)$. This stems from the fact that such an element exists for the symmetric domain $D^{\prime}$ and that it extends naturally to an element of $\mathfrak{g}_{0}^{*}$.

TheOREM 9. Let $D$ be a homogeneous Siegel domain in $\mathscr{V}^{\mathbf{C}} \times \mathscr{U}$ and $\mathfrak{f}$ the isotropy algebra at a point $b \in D$. Then $D$ is symmetric if and only if

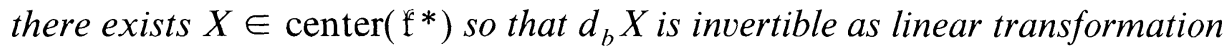
on $T_{b}\left(\mathscr{V}^{\mathrm{C}} \times \mathscr{U}\right) \cong \mathscr{V}^{\mathrm{C}} \times \mathscr{U}$.

Proof. We may assume without restriction $z=i e$. If $D$ is symmetric then we choose $X$ so that $d_{i e} X$ is the complex structure on $\mathscr{V}^{\mathbf{C}} \times \mathscr{U}$. Assume now we have $X \in \operatorname{center}(\mathfrak{f})$ so that $d_{i e} X$ is invertible in $\mathscr{V}^{\mathrm{C}} \times \mathscr{U}$. Then from (66) we derive $\mathscr{X} \ominus \mathscr{P}_{1}=0$ (otherwise the identity of $\mathscr{X} \ominus \mathscr{P}_{1}$ would be annihiliated by $d_{i e} X$ ). This says that $D$ is quasisymmetric. We may assume that $D$ is irreducible. If $D$ is not symmetric then $\mathscr{P}_{1}=0$, a contradiction. This proves the theorem. 


\section{Examples.}

\subsection{Consider the Vinberg cones}

$\Omega=\{(M, N): M, N$ real, $2 \times 2$, symmetric positive definite matrices $\}$ $\Omega^{\prime}=\{M: M$ is real, $3 \times 3$, symmetric positive definite matrix

$$
\text { with } \left.M_{3}^{2}=M_{2}^{3}=0\right\} \text {. }
$$

Imbed these as open subset of $\mathbf{R}^{5}$ so that $\left(X^{k}\right) \in \mathbf{R}^{5}$ corresponds to

$$
\left(\left(\begin{array}{ll}
X^{1} & X^{2} \\
X^{2} & X^{3}
\end{array}\right),\left(\begin{array}{cc}
X^{1} & X^{4} \\
X^{4} & X^{5}
\end{array}\right)\right) \in \Omega, \quad\left(\begin{array}{ccc}
X^{1} & X^{2} & X^{4} \\
X^{2} & X^{3} & 0 \\
X^{4} & 0 & X^{5}
\end{array}\right) \in \Omega^{\prime} .
$$

These cones are dual with respect to the inner product

$$
X \cdot Y=X^{1} Y^{1}+2 X^{2} Y^{2}+X^{3} Y^{3}+2 X^{4} Y^{4}+X^{5} Y^{5} .
$$

We identify the group of complex affine transformations of $\mathbf{C}^{5}$ with the subgroup

$$
\left\{\left(\begin{array}{cc}
A & \tau \\
0 & 1
\end{array}\right): A \in \mathrm{GL}(5, \mathbf{C}), \tau \in \mathbf{C}^{5}\right\}
$$

and similarly for the Lie algebras. Let $\tau_{l} \in \mathbf{C}^{5}$ be the column vector with 1 in the $l$ th position and 0 elsewhere and $E_{m} \in \mathfrak{g l}(5, \mathbf{C})$ the matrix with 1 in the $l$ th row, $m$ th column, 0 elsewhere.

4.1.1. Let $D$ be the tube domain over $\Omega$. Let

$$
\begin{aligned}
X_{1} & =\left(\begin{array}{ll}
0 & \tau_{3} \\
0 & 0
\end{array}\right), \quad X_{2}=\left(\begin{array}{ll}
0 & \tau_{5} \\
0 & 0
\end{array}\right), \quad X_{3}=\left(\begin{array}{ll}
0 & \tau_{1} \\
0 & 0
\end{array}\right) \\
Y_{13} & =\left(\begin{array}{ll}
0 & \tau_{2} \\
0 & 0
\end{array}\right), \quad Y_{23}=\left(\begin{array}{ll}
0 & \tau_{4} \\
0 & 0
\end{array}\right) \\
H_{1} & =\left(\begin{array}{cc}
\frac{1}{2} E_{22}+E_{33} & 0 \\
0 & 0
\end{array}\right), \quad H_{2}=\left(\begin{array}{cc}
\frac{1}{2} E_{44}+E_{55} & 0 \\
0 & 0
\end{array}\right), \\
H_{3} & =\left(\begin{array}{cc}
E_{11}+\frac{1}{2} E_{22}+\frac{1}{2} E_{44} & 0 \\
0 & 0
\end{array}\right) \\
Z_{13} & =\left(\begin{array}{cc}
E_{21}+2 E_{32} & 0 \\
0 & 0
\end{array}\right), \quad Z_{23}=\left(\begin{array}{cc}
E_{41}+2 E_{54} & 0 \\
0 & 0
\end{array}\right) .
\end{aligned}
$$

These elements form a basis for a normal $j$-algebra $(\mathfrak{Z}, j)$ associated to $D$. Here $j\left(X_{k}\right)=H_{k}, j\left(Y_{k l}\right)=Z_{k l}, a=\operatorname{span}\left\{H_{1}, H_{2}, H_{3}\right\}$. If $\varepsilon_{k}$ is defined on 
a by $\varepsilon_{k}\left(H_{l}\right)=\delta_{k l}$ for $k, l=1,2,3$, then the root spaces are

$$
\begin{gathered}
\mathfrak{n}_{\varepsilon_{k}}=\mathbf{R} X_{k}, \quad k=1,2,3 \quad \mathfrak{n}_{\left(\varepsilon_{k}+\varepsilon_{3}\right) / 2}=\mathbf{R} Y_{k 3}, \\
\mathfrak{n}_{\left(\varepsilon_{k}-\varepsilon_{3}\right) / 2}=\mathbf{R} Z_{k 3}, \quad k=1,2 .
\end{gathered}
$$

Since the root spaces are all one dimensional, (46) shows that the space $\delta$ of skew-symmetric derivations is trivial. From [3], we find that, up to constant multiple, the form $\omega$ defining the Bergman metric is given by

$$
\omega\left(X_{1}\right)=\omega\left(X_{2}\right)=3 / 2, \omega\left(X_{3}\right)=2 \text {. }
$$

Since $\omega\left(X_{1}\right) \neq \omega\left(X_{3}\right), \omega\left(X_{2}\right) \neq \omega\left(X_{3}\right)$, (3.7) shows $\mathfrak{n}_{\left(\varepsilon_{1} \pm \varepsilon_{3}\right) / 2} \oplus \mathfrak{n}_{\left(\varepsilon_{2} \pm \varepsilon_{3}\right) / 2}$ $\subset \mathfrak{n}_{2}$ (in the notation of Theorem 3). Then (3.6) implies also $\mathfrak{n}_{\varepsilon_{1}} \oplus \mathfrak{n}_{\varepsilon_{2}} \subset$ $n_{2}$. Now one can check directly that $\nabla_{X_{3}} R=0$ and $C_{X}\left(\nabla_{X_{3}}\right) \in \mathbf{R} \nabla_{X_{3}}$ for all $X \in \mathfrak{S}$ to conclude from (2.8) that $\lambda(\mathfrak{f})=\mathbf{R} \nabla_{X_{3}}$ (alternatively, from other computations, one knows that $\operatorname{dim}$ Aut $\left.D>\operatorname{dim}_{\mathbf{R}} D\right)$. In any case, one now gets

$$
\begin{gathered}
\mathfrak{n}_{1}=\mathfrak{n}_{\varepsilon_{3}}, \quad \mathfrak{n}_{2}=\mathfrak{n}_{\varepsilon_{1}} \oplus \mathfrak{n}_{\varepsilon_{2}} \oplus \mathfrak{n}_{\left(\varepsilon_{1} \pm \varepsilon_{3}\right) / 2} \oplus \mathfrak{n}_{\left(\varepsilon_{2} \pm \varepsilon_{3}\right) / 2} \\
\mathfrak{a}_{1}=\mathbf{R} H_{3}, \quad \mathfrak{a}_{2}=\mathbf{R} H_{1} \oplus \mathbf{R} H_{2}
\end{gathered}
$$

and $\operatorname{dim} \mathfrak{f}=1$.

By the proof of Theorem 7 and the preceding Remark, we know that any vector field $Y \in a_{2} \oplus j a_{2}$ is annihilated by the infinitesimal isotropy representation and so is $G$-invariant. Direct computation shows that $a_{2} \oplus j \mathfrak{a}_{2}$ is the full subspace of $\mathfrak{g}$ annihiliated by $\lambda(\mathfrak{f})$.

In this case, one can easily compute the isotropy representation on the group level, namely $\Lambda(K)=\left\{e^{t \nabla x_{3}}: t \in \mathbf{R}\right\}$ leaves invariant each space $\mathfrak{n}_{\alpha} \oplus j \mathfrak{n}_{\alpha}$ (for each nonzero root $\alpha$ ) and acts there by

$$
e^{t \nabla x_{3} \mid\left(\mathfrak{n}_{\alpha} \oplus j \mathfrak{n}_{\alpha}\right)=\left\{\left(\cos t\left|\alpha\left(H_{3}\right)\right|\right) I+\left(\sin t\left|\alpha\left(H_{3}\right)\right|\right) j\right\} .}
$$

4.1.2. Now let $D$ be the tube domain over the dual cone $\Omega^{\prime}$. A very similar computation shows that there is an associated normal $j$-algebra $\mathfrak{g}$ of rank 3 (i.e. $\operatorname{dim} a=3$ ) and the root spaces are again all one dimensional but now have the form

$$
\mathfrak{n}_{\varepsilon_{k}}, \quad k=1,2,3 ; \quad \mathfrak{n}_{\left(\varepsilon_{1} \pm \varepsilon_{l}\right) / 2}, \quad l=2,3 .
$$

As before, the space $\delta$ of skew-symmetric derivations is trivial. However, when we now compute (from [3]) the form $\omega$ giving the Bergman metric, we get, up to constant multiple,

$$
\omega\left(X_{1}\right)=2, \quad \omega\left(X_{2}\right)=\omega\left(X_{3}\right)=3 / 2 .
$$

Since $\omega\left(X_{1}\right) \neq \omega\left(X_{2}\right), \omega\left(X_{1}\right) \neq \omega\left(X_{3}\right)$, we conclude as before that $\mathfrak{n}_{\left(\varepsilon_{1} \pm \varepsilon_{2}\right) / 2} \oplus \mathfrak{n}_{\left(\varepsilon_{1} \pm \varepsilon_{3}\right) / 2} \subset \mathfrak{n}_{2}$ and hence also $\mathfrak{n}_{\varepsilon_{1}} \subset \mathfrak{n}_{2}$. Direct computation shows that $\nabla_{X_{2}} R=\nabla_{X_{3}} R=0$ and $C_{X}\left(\mathbf{R} \nabla_{X_{2}} \oplus \mathbf{R} \nabla_{X_{3}}\right) \subset \mathbf{R} \nabla_{X_{2}} \oplus \mathbf{R} \nabla_{X_{3}}$ 
for all $X \in \mathfrak{S}$. Thus

$$
\begin{gathered}
\mathfrak{n}_{1}=\mathfrak{n}_{\varepsilon_{2}} \oplus \mathfrak{n}_{\varepsilon_{3}}, \quad \mathfrak{n}_{2}=\mathfrak{n}_{\varepsilon_{1}} \oplus \mathfrak{n}_{\left(\varepsilon_{1} \pm \varepsilon_{2}\right) / 2} \oplus \mathfrak{n}_{\left(\varepsilon_{1} \pm \varepsilon_{3}\right) / 2} \\
\mathfrak{a}_{1}=\mathbf{R} H_{2} \oplus \mathbf{R} H_{3}, \quad \mathfrak{a}_{2}=\mathbf{R} H_{1}
\end{gathered}
$$

and $\operatorname{dim} \mathfrak{f}=2$.

One can compute the Szegö kernel $S(z, z)$ (up to constant multiple) by knowing its invariance properties under $\operatorname{Aff}(D)$. In this case, letting $z=\left(z^{k}\right)$ be the natural complex coordinate on $\mathbf{C}^{5}$, one gets that the Szegö kernel is a constant multiple of

$$
\left(z^{3}-\bar{z}^{3}\right)^{1 / 2}\left(z^{5}-\bar{z}^{5}\right)^{1 / 2}(\operatorname{det}(Z-\bar{Z}))^{-2}
$$

where $Z$ is the $3 \times 3$ matrix corresponding to $z$. Let $\Delta$ be the Laplacian of the Bergman metric and $P_{\eta}(z)$ the Poisson kernel. A straightforward but messy calculation shows that $\left(\Delta P_{\eta}\right)(i I)=0$ for $\eta=\left(\eta^{1}, 0, \eta^{3}, 0, \eta^{5}\right)^{t}$ (and possibly for other values of $\eta$ in the Silov boundary). In this case, the action of $\exp \left(a X_{1}+b X_{2}+c X_{3}\right)$ on $\mathbf{C}^{5}$ is translation by the constant vector $(a, 0, b, 0, c)^{t}$. In fact, one can compute that $K \cdot 0=P_{1}=\left\{\eta \in \mathbf{R}^{5}\right.$ : $\left.\eta=\left(0,0, \eta^{3}, 0, \eta^{5}\right)^{t}\right\}$ so $B_{p}$ is certainly larger than $K \cdot 0$ (cf. Theorem 6 and the following remark).

4.2. Let $D$ be Pyatetskii-Shapiro's 8 (real)-dimensional example. As computed in [3], an associated normal $j$-algebra $\mathfrak{g}$ is of rank 2 (so $\operatorname{dim} a=2$ ) with root spaces $\mathfrak{n}_{\varepsilon_{k}}, k=1$, , of dimension $1: \mathfrak{n}_{\varepsilon_{1} / 2}$ of (real) dimension $2 ; \mathfrak{n}_{\left(\varepsilon_{1} \pm \varepsilon_{2}\right) / 2}$ of dimension 1 . Since all root spaces except $\mathfrak{n}_{\varepsilon_{1} / 2}$ are one dimensional, every skew-symmetric derivation is trivial except possibly on $\mathfrak{n}_{\varepsilon_{1} / 2}$. It is easy to check that the algebra of skew-symmetric derivations is

$$
\grave{D}=\mathbf{R}\left(j \mid \mathfrak{n}_{\varepsilon_{1} / 2}\right) .
$$

Computing the form $\omega$ definining the Bergman metric as in [3], we get (up to constant multiple)

$$
\omega\left(X_{1}\right)=1, \quad \omega\left(X_{2}\right)=3 / 4 \text {. }
$$

Since $\omega\left(X_{1}\right) \neq \omega\left(X_{2}\right),(3.4)-(3.7)$ imply that

$$
\mathfrak{n}_{\varepsilon_{1} / 2} \oplus \mathfrak{n}_{\varepsilon_{1}} \oplus \mathfrak{n}_{\left(\varepsilon_{1} \pm \varepsilon_{2}\right) / 2} \subset \mathfrak{n}_{2} .
$$

Direct computation shows that $\left(\nabla_{X_{2}} R\right)\left(\mathfrak{n}_{\left(\varepsilon_{1}+\varepsilon_{2}\right) / 2}, \mathfrak{n}_{\varepsilon_{1} / 2}\right) \mathfrak{n}_{\left(\varepsilon_{1}+\varepsilon_{2}\right) / 2} \neq 0$ so we also get $n_{\varepsilon_{2}} \subset \mathfrak{n}_{2}$. Thus

$$
\begin{array}{ll}
\mathfrak{n}_{1}=0, & \mathfrak{n}_{2}=\mathfrak{n} \\
\mathfrak{a}_{1}=0, & \mathfrak{a}_{2}=\mathfrak{a} .
\end{array}
$$

Again, $\operatorname{dim} \mathfrak{f}=1$. 
Since $\lambda(\mathfrak{f})=\mathbf{R}\left(j \mid \mathfrak{n}_{\varepsilon_{1} / 2}\right)$, the subspace of $\mathfrak{g}$ annihilated by $\lambda(\mathfrak{f})$ is the 6-dimensional space $\left(\mathfrak{n}_{\varepsilon_{1} / 2}\right)^{\perp}$. This gives the $G$-invariant vector fields, as in Theorem 7. The proof of Theorem 7 only guarantees that the 4-dimensional space $a_{2} \oplus j a_{2}$ is $G$-invariant.

4.3. Let $\mathscr{V}$ be the space of real symmetric $2 \times 2$ matrices and $\Omega$ the cone of positive definite matrices in $\mathscr{V}$. Let $\mathscr{U}=\mathrm{C}^{2}, F(U, V)=$ $\frac{1}{2}\left(U \bar{V}^{t}+\bar{V} U^{t}\right)$ and $D$ the corresponding Siegel domain. This is example $B_{2}$ (with $r=2, s=1$ ) of [13] and Example C II (with $m=2$ ) of [27] where however there are errors in the computation of $g_{1}$. The example is known to be quasisymmetric nonsymmetric homogeneous so $\mathfrak{g}_{1 / 2}=\mathfrak{g}_{1}=$ $0 ;$ by $\left[13\right.$, p. 41] one finds $\operatorname{dim} \mathfrak{g}_{0}=5$. Thus $\operatorname{dim} \mathfrak{f}=\operatorname{dim} \mathfrak{f}_{0}=\operatorname{dim} \mathfrak{g}_{0}-$ $\operatorname{dim} \mathscr{V}=2$.

The corresponding normal $j$-algebra $\mathfrak{g}$ has $\operatorname{rank}=\operatorname{dim} \mathfrak{a}=2$ with root spaces $\mathfrak{n}_{\varepsilon_{k}}, k=1,2$, of dimension $1 ; \mathfrak{n}_{\varepsilon_{k} / 2}, k=1,2$ of (real) dimension 2; $\mathfrak{n}_{\left(\varepsilon_{1} \pm \varepsilon_{2}\right) / 2}$ of dimension 1. By the Remark after Theorem 5, $j \mid\left(\mathfrak{n}_{\varepsilon_{1} / 2} \oplus \mathfrak{n}_{\varepsilon_{2} / 2}\right)$ is a skew-symmetric derivation. Using bracket product relations between $\mathfrak{n}_{\varepsilon_{1} / 2}$ and $\mathfrak{n}_{\varepsilon_{2} / 2}$ and the fact that the other root spaces are one dimensional, one gets

$$
\mathfrak{D}=\mathbf{R}\left(j \mid\left(\mathfrak{n}_{\varepsilon_{1} / 2} \oplus \mathfrak{n}_{\varepsilon_{2} / 2}\right)\right) .
$$

Since we are only missing one dimension in $\mathfrak{f}$, relations (3.3)-(3.6) show that

$$
\begin{gathered}
\mathfrak{n}_{1}=\mathfrak{n}_{\left(\varepsilon_{1}-\varepsilon_{2}\right) / 2}, \quad \mathfrak{n}_{2}=\mathfrak{n}_{\varepsilon_{1}} \oplus \mathfrak{n}_{\varepsilon_{2}} \oplus \mathfrak{n}_{\varepsilon_{1} / 2} \oplus \mathfrak{n}_{\varepsilon_{2} / 2} \oplus \mathfrak{n}_{\left(\varepsilon_{1}+\varepsilon_{2}\right) / 2} \\
a_{1}=\mathbf{R} j\left(X_{1}-X_{2}\right), \quad \mathfrak{a}_{2}=\mathbf{R} j\left(X_{1}+X_{2}\right) .
\end{gathered}
$$

Note that in this case, (3.7) gives no information because $\omega\left(X_{1}\right)=\omega\left(X_{2}\right)$, (which also follows from $a_{1} \perp a_{2}$ ).

In this example, if we take any deformation of the Bergman metric by using $\omega\left(X_{1}\right)=1, \omega\left(X_{2}\right)=t$, we get a one parameter family of Kähler metrics. In all cases, we still have $\delta=\mathbf{R}\left(j \mid\left(n_{\varepsilon_{1} / 2} \oplus n_{\varepsilon_{2} / 2}\right)\right)$ but (3.7) now implies $\mathfrak{g}_{1}=\mathfrak{n}_{1}=0$ for $t \neq 1$. Thus we have a jump in the dimension of $\mathfrak{f}$ at $t=1$ (the Bergman metric).

Returning to the Bergman metric, one computes that the space annihilated by $\lambda(\mathfrak{f})=\mathfrak{d} \oplus \nabla_{\mathfrak{n}_{\left(\mathfrak{q}_{1}-\varepsilon_{2}\right) / 2}}$ is exactly $\mathfrak{a}_{2} \oplus j \mathfrak{a}_{2}$, the minimum possible by the proof of Theorem 7 .

\section{REFERENCES}

[1] R. Azencott and E. Wilson, Homogeneous manifolds with negative curvature I, Trans. Amer. Math. Soc., 215 (1976), 323-362.

[2] Homogeneous manifolds with negative curvature II, Mem. Amer. Math. Soc., 8, Number 178, (1976). 
[3] J. E. D'Atri, The curvature of homogeneous Siegel domains, J. Differential Geom., 15 (1980), 61-70.

[4] Holomorphic sectional curvatures of bounded homogeneous domains and related questions, Trans. Amer. Math. Soc., 256 (1979), 405-413.

[5] _ Sectional curvatures and quasi-symmetric domains, J. Differential Geom., 16 (1981), 11-18.

[6] J. E. D'Atri and I. Dotti Miatello, A characterization of bounded symmetric domains by curvature, Trans. Amer. Math. Soc., 276 (1983), 531-540.

[7] J. Dorfmeister, Zur Kunstruktion homogener Kegel, Math. Ann., 216 (1975), 79-96.

[8] _ Inductive construction of homogeneous cones, Trans. Amer. Math. Soc., 252 (1979), 321-349.

[9] Algebraic description of homogeneous cones, Trans. Amer. Math. Soc., 255 (1979), 61-89.

[10] Peirce-Zerlegungen und Jordan-Strukturen zu homogenen Kegeln, Math. Z., 169 (1979), 179-194.

[11] _ Quasi symmetric Siegel domains and the automorphisms of homogeneous Siegel domains, Amer. J. Math., 102 (1980), 537-563.

[12] __ Homogeneous Siegel domains, Nagoya Math. J., 86 (1982), 39-83.

[13] ___ Homogene Siegel-Gebiete, Habilitationsschrift, Münster, 1978.

[14] __ Simply transitive groups and Kähler structures on homogeneous Siegel domains, to appear in Trans. Amer. Math. Soc.

[15] __ Homogeneous Kähler manifolds admitting a transitive solvable group of automorphisms, to appear in Ann. Sci. École Norm. Sup.

[16] S. G. Gindikin, Analysis in homogeneous domains, Uspekhi Mat. Nauk, 19 (1964), 3-92.

[17] S. G. Gindikin, I. I. Pyatetskii-Shapiro and E. B. Vinberg, Homogeneous Kähler manifolds, in "Geometry of homogeneous domains", C.I.M.E. (1967), 1-88.

[18] S. Helgason, Differential Geometry and Symmetric Spaces, Academic Press, N. Y., 1962.

[19] S. Kaneyuki, Homogeneous Bounded Domains and Siegel Domains, Lecture Notes in Math, V. 241, Springer, Berlin, 1971.

[20] W. Kaup, Einige Bemerkungen über polynomiale Vektorfelder, Jordanalgebren, und die Automorphismen von Siegelschen Gebieten, Math. Ann., 204 (1973), 131-144.

[21] W. Kaup, Y. Matsushima, T. Ochiai, On the automorphism and equivalences of generalized Siegel domains, Amer. J. Math., 92 (1970), 475-497.

[22] S. Kobayashi, Transformation Groups in Differential Geometry, Springer, Berlin, 1972.

[23] A. Koranyi, The Poisson integral for generalized half-planes and bounded symmetric domains, Ann. of Math., 82 (1965), 332-350.

[24] A. Koranyi and E. Stein, $H^{2}$ spaces of generalized half-spaces, Studia Math., 44 (1972), 379-388.

[25] J. L. Koszul, Sur la forme hermitienne canonique des espaces homogenes complexes, Canad. J. Math., 7 (1955), 562-576.

[26] O. Loos, Jordan Pairs, Lectures Notes in Math., V. 460, Springer, Berlin, 1973.

[27] S. Murakami, On Automorphisms of Siegel Domains, Lecture Notes in Math., V. 286, Springer, Berlin, 1971.

[28] I. I. Pyatetskii-Shapiro, Automorphic Functions and the Geometry of Classical Domains, English edition, Gordon and Breach, New York, 1969.

[29] O. S. Rothaus, Domains of positivity, Abh. Math Sem. Univ. Hamburg, 24 (1960), 189-235.

[30] E. B. Vinberg, The Theory of Convex Homogeneous Cones, English translation, Trans. Moscow Math. Soc., 12 (1963), 340-403. 
[31] The Structure of the Group of Automorphisms of a Homogeneous convex cone, English translation, Trans. Moscow Math. Soc., 13 (1965), 63-93.

[32] H. L. Williams, The structure of isometry groups for a class of homogeneous Riemannian manifolds with non-positive curvature, $\mathrm{Ph}$. D. thesis, Washington Univ., St. Louis, 1978.

[33] Xu Yichao, On the Bergman kernel function of homogeneous bounded domains, Scientia Sinica, Special Issue (II), (1979), 80-90.

Received October 15, 1983.

RUTGERS UNIVERSITY

NEW BRUNSWICK, NJ 08903

UNIVERSITY OF GEORGIA

Athens, GA 30602

AND

HONGZHOU UNIVERSITY

Zhejiang, Peoples Republic of China 


\section{PACIFIC JOURNAL OF MATHEMATICS EDITORS}

\author{
V. S. VARADARAJAN (Managing Editor) \\ University of California \\ Los Angeles, CA 90024 \\ Charles R. DePrima \\ California Institute of Technology \\ Pasadena, CA 91125 \\ R. FINN \\ Stanford University \\ Stanford, CA 94305
}

\author{
HeRmanN FlaschKa \\ University of Arizona \\ Tucson, AZ 857.21
}

RAMESH A. GANGOLli

University of Washington

Seattle, WA 98195

ROBION KIRBY

University of California

Berkeley, CA 94720
C. C. MOORE

University of California

Berkeley, CA 94720

H. SAMELSON

Stanford University

Stanford, CA 94305

HAROLD STARK

University of California, San Diego

La Jolla, CA 92093

\section{ASSOCIATE EDITORS}
R. ARENS
E. F. BECKENBACH
B. H. NeumanN
F. WOLF
K. YoSHIDA
(1906-1982)

\section{SUPPORTING INSTITUTIONS}

\begin{abstract}
UNIVERSITY OF ARIZONA
UNIVERSITY OF BRITISH COLUMBIA

CALIFORNIA INSTITUTE OF TECHNOLOGY

UNIVERSITY OF CALIFORNIA

MONTANA STATE UNIVERSITY

UNIVERSITY OF NEVADA, RENO

NEW MEXICO STATE UNIVERSITY

OREGON STATE UNIVERSITY
\end{abstract}

\author{
UNIVERSITY OF OREGON \\ UNIVERSITY OF SOUTHERN CALIFORNIA \\ STANFORD UNIVERSITY \\ UNIVERSITY OF HAWAII \\ UNIVERSITY OF TOKYO \\ UNIVERSITY OF UTAH \\ WASHINGTON STATE UNIVERSITY \\ UNIVERSITY OF WASHINGTON
}

The Supporting Institutions listed above contribute to the cost of publication of this Journal, but they are not owners or publishers and have no responsibility for its content or policies.

Mathematical papers intended for publication in the Pacific Journal of Mathematics should be in typed form or offset-reproduced (not dittoed), double spaced with large margins. Please do not use built up fractions in the text of the manuscript. However, you may use them in the displayed equations. Underline Greek letters in red, German in green, and script in blue. The first paragraph must be capable of being used separately as a synopsis of the entire paper. In particular it should contain no bibliographic references. Please propose a heading for the odd numbered pages of less than 35 characters. Manuscripts, in triplicate, may be sent to any one of the editors. Please classify according to the scheme of Math. Reviews, Index to Vol. 39. Supply name and address of author to whom proofs should be sent. All other communications should be addressed to the managing editor, or Elaine Barth, University of California, Los Angeles, California 90024.

There are page-charges associated with articles appearing in the Pacific Journal of Mathematics. These charges are expected to be paid by the author's University, Government Agency or Company. If the author or authors do not have access to such Institutional support these charges are waived. Single authors will receive 50 free reprints; joint authors will receive a total of 100 free reprints. Additional copies may be obtained at cost in multiples of 50 .

The Pacific Journal of Mathematics is issued monthly as of January 1966. Regular subscription rate: $\$ 190.00$ a year (5 Vols., 10 issues). Special rate: $\$ 66.00$ a year to individual members of supporting institutions.

Subscriptions, orders for numbers issued in the last three calendar years, and changes of address should be sent to Pacific Journal of Mathematics, P.O. Box 969, Carmel Valley, CA 93924, U.S.A. Old back numbers obtainable from Kraus Periodicals Co., Route 100, Millwood, NY 10546.

The Pacific Journal of Mathematics at P.O. Box 969, Carmel Valley, CA 93924 (ISSN 0030-8730) publishes 5 volumes per year. Application to mail at Second-class postage rates is pending at Carmel Valley, California, and additional mailing offices. Postmaster: Send address changes to Pacific Journal of Mathematics, P.O. Box 969, Carmel Valley, CA 93924.

PUBLISHED BY PACIFIC JOURNAL OF MATHEMATICS, A NON-PROFIT CORPORATION

Copyright $\odot 1985$ by Pacific Journal of Mathematics 


\section{Pacific Journal of Mathematics}

Vol. 120, No. $2 \quad$ October, 1985

Philip Marshall Anselone and Mike Treuden, Regular operator

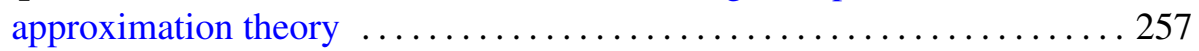

Giuseppe Baccella, Semiprime $\aleph-Q F 3$ rings $\ldots \ldots \ldots \ldots \ldots \ldots \ldots \ldots . \ldots \ldots$

Earl Robert Berkson and Thomas Alastair Gillespie, The generalized M.

Riesz theorem and transference $\ldots \ldots \ldots \ldots \ldots \ldots \ldots \ldots . \ldots 279$

Joachim Boidol, A Galois-correspondence for general locally compact

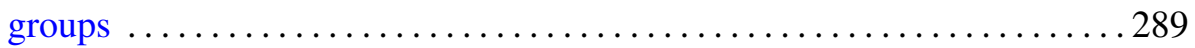

Joseph Eugene D'Atri, Josef Dorfmeister and Yan Da Zhao, The isotropy

representation for homogeneous Siegel domains ............... 295

C. Debiève, On Banach spaces having a Radon-Nikodým dual

Michael Aaron Freedman, Existence of strong solutions to singular

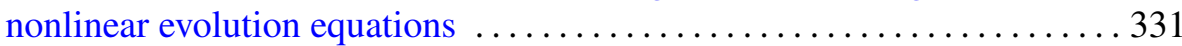

Francisco Jose Freniche, Grothendieck locally convex spaces of continuous

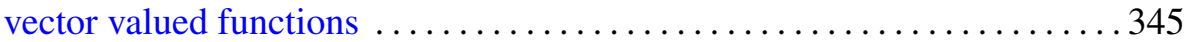

Hans-Peter Künzi and Peter Fletcher, Extension properties induced by complete quasi-uniformities . ............................ 357

Takaŝi Kusano, Charles Andrew Swanson and Hiroyuki Usami, Pairs of

positive solutions of quasilinear elliptic equations in exterior domains . . 385

Angel Rafael Larotonda and Ignacio Zalduendo, Spectral sets as Banach

manifolds

J. Martínez-Maurica and C. Pérez García, A new approach to the

Kreı̆n-Milman theorem

Christian Pommerenke, On the boundary continuity of conformal maps . . . 423

M. V. Subba Rao, Some Rogers-Ramanujan type partition theorems

Stephen Edwin Wilson, Bicontactual regular maps .........

Jaap C. S. P. van der Woude, Characterizations of (H)PI extensions

Kichoon Yang, Deformation of submanifolds of real projective space

Subhashis Nag, Errata: "On the holomorphy of maps from a complex to a

real manifold" 